\title{
Microscopic description of low-lying one- and two-phonon states and application to ${ }^{96} \mathrm{Zr}$
}

\author{
S. A. Fayans ${ }^{1}$ \\ GANIL, B.P. 5027, F-14021 Caen Cedex, France \\ A. P. Platonov \\ Russian Research Centre - Kurchatov Institute, Moscow 123182, Russia \\ G. Graw and D. Hofer \\ Sektion Physik der Universität München, 85748 Garching, FRG
}

\begin{abstract}
:
A self-consistent approach to finite Fermi systems is expanded for the description of two-phonon states in non-magic nuclei with weak anharmonicity. It is based on a density-functional approach, the quasi-particle random-phase approximation (QRPA) and the Green's function method, the latter enabling one to calculate the diagrams of the phonon-phonon interaction in the coordinate representation. This approach is applied to the low-lying excitations in ${ }^{96} \mathrm{Zr}$. The transition density of the two-phonon state $\left[3_{1}^{-} \otimes 3_{1}^{-}\right]_{6+}$ in ${ }^{96} \mathrm{Zr}$ is calculated. It is shown that interplay of one-phonon and two-phonon configurations may lead to the origin of two $6^{+}$states with strongly different excitation probabilities. Mixing coefficients and energy splitting of these states are estimated microscopically. The transition probabilities of the one-phonon states $3_{1}^{-}$and $6^{+}$and the mixing coefficients of the one- and two-phonon $6^{+}$states resulting from the microscopic calculations are used as input for DWBA and Coupled-Channels calculations for inelastic scattering of $22 \mathrm{MeV}$ polarized deuterons. Calculated cross sections for the $6^{+}$states are compared with experimental data.
\end{abstract}

PACS numbers: 21.60 Er, 21.60 Jz, 24.10 Eq, 25.45 De, 27.60+j

${ }^{1}$ Permanent address: Kurchatov Institute, Moscow 123182, Russia. 


\section{Introduction}

In recent years, a qualitatively new level has been achieved in studying the lowenergy properties of nuclei, providing access to detailed spectroscopic information and important characteristics of low-lying collective states as their transition densities. For example, in experiments with polarized deuterons ${ }^{1}$ ), a resolution of $5 \mathrm{keV}$ has been attained for the excitation energy region up to $4.5 \mathrm{MeV}$. At a number of accelerators (NIKHEF, MIT, Saclay), precision measurements of charge transition densities were performed through inelastic electron scattering by nuclei with a typical energy resolution near $\delta E / E \approx 10^{-4}$. A comparison of those experimental data with theoretical calculations allows to test various theoretical models and to extract important information on fundamental properties of nuclei.

The analyses of spherical even-even nuclei show the distribution of low-lying collective transition strength and establish in some detail the presence of double-quadrupole two-phonon states, provide some information on quadrupole-octupole two-phonon states. and aim to establish the presence of double-octupole two-phonon excitations from the ground-state. An admixture of these two-phonon configurations with one-phonon excitations often plays an important role. The QRPA calculations of Rosso et al. ${ }^{2}$ ) for ${ }^{96} \mathrm{Zr}$ describe very nicely the one phonon collective and the single particle excitations of this nucleus. However, they do not include the possibility of two phonon excitations.

A distinctive feature of the approach outlined in the present paper and used in first calculations to discuss properties of double-octupole and one-phonon $6^{+}$excitations in ${ }^{96} \mathrm{Zr}$ is a self-consistent description of the ground state and of the excited states, with allowance for pairing correlations. Accordingly, the full computational scheme is divided into three steps: (1) the construction of a single-particle potential and of a quasiparticle basis, (2) the solution of the QRPA-type equations and the derivation of 
transition densities of collective "one-phonon" excited states and (3) the calculation of phonon-phonon interaction diagrams using single-particle Green's functions constructed in the coordinate representation ${ }^{3,4}$ ). The latter point is important since by using coordinate representation ${ }^{5}$ ) one consistently takes into account the contribution of the continuum and avoids truncation of the single-particle basis in the particle-hole channel. In accordance with this scheme the following three sections of the present paper are built up. Section 5 is devoted to the analysis of specific numerical results and to the comparison of these results with other calculations and with experimental observations. In section 6 the conclusions of the preceeding discussions are summarized. Technical aspects are presented in the appendices $\mathrm{A}$ to $\mathrm{C}$.

\section{The self-consistent potential and the quasi-particle basis}

For a self-consistent description of excited states in nonmagic nuclei one first has to obtain the mean fields for neutrons and protons incorporating pairing correlations. For this purpose we use the density-functional method based on the Hohenberg-Kohn theorem ${ }^{6}$ ) and the Kohn-Sham quasiparticle formalism ${ }^{7}$ ), the latter being characterized by the quasiparticle Hamiltonian with a free kinetic energy operator, equating thus the effective nucleon mass $m^{*}$ of such quasiparticles to the bare mass $m\left(m^{*} / m=1\right)$. The nucleon normal density $\rho$ and the anomalous density $\nu$, the latter resulting from pairing correlations, are represented in this approach by the sum of single-particle contributions, i.e., by the shell sums which minimize the energy of the system. The quasiparticle spectrum and the wave functions are calculated in a self-consistent mean field and pairing potential which both are the first functional derivatives of the density functional with respect to the corresponding density. As the effective mass of a nucleon on the Fermi surface in nuclei is close to the bare mass, one may assume to obtain calcu- 
lated quasiparticle levels close to the observed ones. This is essential to describe the low-lying collective states in even-even nuclei, especially those of collective particle-hole nature. These states are treated within the quasiparticle RPA (QRPA) approach with the effective interactions being the second functional derivatives of the energy-density functional. In fact, this method is a version of the self-consistent finite Fermi systems (FFS) theory ${ }^{8}$ ) which has much in common with the Hartree-Fock (HF) method with effective forces ${ }^{9}$ ). The main problem of these approaches in practical application is the choice of the form and of the parametrization of an appropriate density functional. Here we shall use a density functional in the form suggested in ref. ${ }^{10}$ ) where the density-dependence is simulated by simple fractional-linear functions and where the surface contributions are related to finite-range forces.

The energy density is written as

$$
\varepsilon=\varepsilon_{\mathrm{kin}}+\varepsilon_{\mathrm{int}}
$$

where the kinetic energy density is given by

$$
\varepsilon_{\mathrm{kin}}=\sum_{\lambda} n_{\lambda} \varphi_{\lambda}^{*}(\vec{r}) \frac{\vec{p}^{2}}{2 m} \varphi_{\lambda}(\vec{r})
$$

Here, $n_{\lambda}$ is the occupation factor of the single-particle level $\lambda, \varphi_{\lambda}$ the corresponding wave function, $\lambda=n l j \mu \tau$ the standard set of single-particle quantum numbers and $m$ the nucleon mass. For spherical nuclei one obtains

$$
\varepsilon_{\mathrm{kin}}=\frac{1}{4 \pi} \sum_{\lambda} n_{\lambda} \frac{\hbar^{2}}{2 m}\left[\left(\frac{d R_{n l j \tau}(r)}{d r}\right)^{2}+\frac{l(l+1)}{r^{2}} R_{n l j \tau}^{2}(r)\right] .
$$

The interaction energy density is represented as 


$$
\varepsilon_{\mathrm{int}}=\varepsilon_{\mathrm{main}}+\varepsilon_{\mathrm{coul}}+\varepsilon_{s l}+\varepsilon_{\text {pair }}
$$

In the first term,

$$
\varepsilon_{\text {main }}=\frac{2}{3} \epsilon_{F}^{0} \rho_{0}\left[a_{+}^{v} x_{+}^{2} f_{+}^{v}+a_{-}^{v} x_{-}^{2} f_{-}^{v}+a_{+}^{s} x_{+} f_{+}^{s} \widetilde{f_{+}^{s} x_{+}}+a_{-}^{s} x_{-} f_{-}^{s} \widetilde{f_{-}^{s} x_{-}}\right],
$$

we isolate volume $(v)$ and surface $(s)$ isoscalar $(+)$ and isovector $(-)$ terms introducing dimensionless densities $x_{ \pm}=\left(\rho_{n} \pm \rho_{p}\right) / 2 \rho_{0}$, where $\rho_{n}$ and $\rho_{p}$ are the neutron and proton densities respectively. The normalizations are with respect to $2 \rho_{0}$, the equilibrium density for symmetric nuclear matter $(N=Z)$ and to $\epsilon_{F}^{0}$, the corresponding Fermi energy. The terms factorize in interaction strength parameters $a_{ \pm}^{v, s}$, squares of the densities, and density-dependent factors which simulate effectively both many-body correlations and many-body forces:

$$
f_{ \pm}^{v}=\frac{1-h_{1 \pm}^{v} x_{+}}{1+h_{2 \pm}^{v} x_{+}}, \quad f_{ \pm}^{s}=\frac{1}{1+h_{ \pm}^{s} x_{+}}
$$

Here the $h$ 's describe the density dependence of the respective strengths. In the surface terms we introduce the contributions from finite range effects defining

$$
\widetilde{f_{ \pm}^{s} x}=\int D\left(\vec{r}-\vec{r}^{\prime}\right) f_{ \pm}^{s}\left(\vec{r}^{\prime}\right) x_{ \pm}\left(\vec{r}^{\prime}\right) \mathrm{d} \vec{r}^{\prime}
$$

with

$$
\begin{aligned}
D\left(\vec{r}-\vec{r}^{\prime}\right)= & V\left(\left|\vec{r}-\vec{r}^{\prime}\right|\right)-\delta\left(\vec{r}-\vec{r}^{\prime}\right) \\
& =\frac{1}{4 \pi R^{2}\left|\vec{r}-\vec{r}^{\prime}\right|} \exp \left(-\frac{\left|\vec{r}-\vec{r}^{\prime}\right|}{R}\right)-\delta\left(\vec{r}-\vec{r}^{\prime}\right),
\end{aligned}
$$


where $R$ is a range parameter. In the momentum representation eq. (2.8) reads as

$$
D(q)=-\frac{(q R)^{2}}{1+(q R)^{2}}
$$

Thus the last two terms in eq. (2.5) vanish in the case of uniform nuclear matter whereas for finite nuclei they correspond to the contributions of the surface isoscalar and isovector potential energies generated by the density-dependent finite-range forces.

The energy density of the Coulomb interaction $\varepsilon_{\text {coul }}$ is taken in usual form ${ }^{9}$ ) including the exchange term in the Slater approximation:

$$
\varepsilon_{\text {coul }}=2 \pi e^{2} \rho_{p}(r)\left(\frac{1}{r} \int_{0}^{r} \rho_{p}(r) r^{2} \mathrm{~d} r+\int_{r}^{\infty} \rho_{p}(r) r \mathrm{~d} r\right)-\frac{3}{4}\left(\frac{3}{\pi}\right)^{1 / 3} e^{2} \rho_{p}^{4 / 3}(r)
$$

The spin-orbit term $\varepsilon_{s l}$ derives from the spin-orbit interactions, $\propto\left(\kappa+\kappa^{\prime} \vec{\tau}_{1}\right.$. $\left.\vec{\tau}_{2}\right)\left[\vec{\nabla}_{1} \delta\left(\vec{r}_{1}-\vec{r}_{2}\right) \times\left(\vec{p}_{1}-\vec{p}_{2}\right)\right] \cdot\left(\vec{\sigma}_{1}+\vec{\sigma}_{2}\right)$, and from velocity spin-dependent interactions, $\propto\left(g_{1}+g_{1}^{\prime} \vec{\tau}_{1} \cdot \vec{\tau}_{2}\right)\left(\vec{\sigma}_{1} \cdot \vec{\sigma}_{2}\right)\left(\vec{p}_{1} \cdot \vec{p}_{2}\right)$. For spherical nuclei these terms can be expressed using spin-orbit densities,

$$
\rho_{s i}^{n, p}(\vec{r})=\sum_{\lambda} n_{\lambda}<\vec{\sigma} \cdot \vec{l}>_{\lambda}\left|\varphi_{\lambda}^{n, p}(\vec{r})\right|^{2}
$$

where $\langle\vec{\sigma} \cdot \vec{l}\rangle_{\lambda}=j(j+1)-l(l+1)-3 / 4$. For the corresponding energy density one obtains

$$
\varepsilon_{s l}=C_{0} r_{0}^{2} \sum_{i, k=n, p}\left(\frac{1}{r} \rho_{s l}^{i} \kappa^{i k} \frac{d \rho^{k}}{d r}+\frac{1}{4 r^{2}} \rho_{s l}^{i} g_{1}^{i k} \rho_{s l}^{k}\right)
$$

where $\kappa^{n n}=\kappa^{p p}=\kappa+\kappa^{\prime}, \quad \kappa^{n p}=\kappa^{p n}=\kappa-\kappa^{\prime} ; g_{1}^{n n}=g_{1}^{p p}=g_{1}+g_{1}^{\prime}, g_{1}^{n p}=$ 
$g_{1}^{p n}=g_{1}-g_{1}^{\prime}$ represent spin-orbit strength parameters normalized to $C_{0}=2 \varepsilon_{F}^{0} / 3 \rho_{0}$, and $r_{0}=\left(3 / 8 \pi \rho_{0}\right)^{1 / 3}$.

The last term in eq. (2.4), the pairing energy density $\varepsilon_{\text {pair }}$, is chosen as

$$
\varepsilon_{\text {pair }}=\frac{1}{2} \nu \mathcal{F}^{\xi} \nu
$$

where $\nu$ is the anomalous nucleon density and $\mathcal{F}^{\xi}$ plays the role of an effective interaction in the particle-particle and hole-hole channels. This force is represented in the simplest form as

$$
\mathcal{F}^{\xi(n n)}=\mathcal{F}^{\xi(p p)}=C_{0} f^{\xi} \delta\left(\vec{r}-\vec{r}^{\prime}\right)
$$

with $f^{\xi}$ being a dimensionless interaction constant of the FFS theory ${ }^{11}$ ). The superscript $\xi$ refers to the energy cutoff parameter which defines the number of single-particle levels taken into account when evaluating the anomalous Green's functions and, correspondingly, when solving the equations for the pairing fields $\Delta(\vec{r})$ and for the chemical potentials $\mu$ as well as for the dynamical FFS equations for excited states.

Thus, the total interaction energy of a superfluid nucleus with normal and anomalous densities $\rho$ and $\nu$ is written as $E_{\text {int }}[\rho, \nu]=\int d \vec{r} \varepsilon_{\text {int }}(\vec{r})$, where $\varepsilon_{\text {int }}(\vec{r})$ is defined as above. The self-consistent calculations with such a functional are similar to the standard variational $\mathrm{HF}-\mathrm{B}$ procedure for the eigenstate problem, $\hat{\mathcal{H}} \hat{\Psi}=E \hat{\Psi}$, in which the singleparticle Hamiltonian takes the form

$$
\hat{\mathcal{H}}=\left(\begin{array}{cc}
h-\mu & -\Delta \\
-\Delta & \mu-h
\end{array}\right)
$$


where

$$
h=\frac{p^{2}}{2 m}+\frac{\delta E_{\mathrm{int}}[\rho, \nu]}{\delta \rho}, \Delta=-\frac{\delta E_{\mathrm{int}}[\rho, \nu]}{\delta \nu} .
$$

These equations were solved iteratively as follows. For given densities $\left(\rho^{(i)}, \nu^{(i)}\right)$, from the above functional the elements of the Hamiltonian $\hat{\mathcal{H}}^{(i)}$ were derived, from the eigenvalues $E^{(i)}$ and wave functions $\hat{\Psi}=\left(u^{(i)}, v^{(i)}\right)$ the new densities $\left(\rho^{(i+1)}, \nu^{(i+1)}\right)$ were calculated from which the new mean fields $(U, \Delta)$ were obtained. For the next iteration superpositions of the previous mean fields and these new ones were used with weights of 0.85 and 0.15 , respectively. In each iteration the chemical potential $\mu^{(i)}$ was derived from the condition that the normal density provides the proper value of the particle number. This procedure was continued until complete convergence was achieved. The parameters of the density functional were chosen from a fit ${ }^{12,13}$ ) of the binding energies, charge distributions, and single-particle spectra of magic nuclei ${ }^{40} \mathrm{Ca},{ }^{48} \mathrm{Ca},{ }^{208} \mathrm{~Pb}$ and of non-magic ones, both with weak superfluidity ( ${ }^{90} \mathrm{Zr},{ }^{146} \mathrm{Gd}$ ) and with developed pairing (even-even $\mathrm{Sn}$ and $\mathrm{Pb}$ isotopes). Pairing was treated in the diagonal approximation on the basis of all bound single-particle levels. To keep the number of parameters in the fitting procedure small it was assumed that in eq. (2.5) $f_{-}^{v}=f_{+}^{v}$ (i.e. $h_{1-}^{v}=h_{1+}^{v}$ and $h_{2-}^{v}=h_{2+}^{v}$ ) and $a_{-}^{s}=0$. As a result, the following set of parameters was deduced:

$$
\begin{gathered}
a_{+}^{v}=-7.391, h_{1+}^{v}=0.037, h_{2+}^{v}=1.322 \\
a_{-}^{v}=3.595 \\
a_{+}^{s}=-10.0, h_{2+}^{s}=0.31 \\
\kappa^{p p}=\kappa^{p n}=0.205 \\
g_{1}^{p p}=-g_{1}^{p n}=-0.11 \\
f^{\xi}=-0.33 \\
R=0.35 \mathrm{fm}
\end{gathered}
$$


We note some relations between a number of these parameters and certain nuclearmatter properties:

$$
\left.\begin{array}{c}
a_{+}^{v}=\alpha+(5 \alpha+6) / 5 \eta, \\
h_{1+}^{v}=1-\alpha / a_{+}^{v} \eta, \\
h_{2+}^{v}=1 / \eta-1, \\
\left(3 \beta^{0} / \epsilon_{F}^{0}-1\right) / f_{-}^{v}\left(x_{+}=1\right),
\end{array}\right\}
$$

where

$$
\alpha=3 \frac{\mu^{0}}{\epsilon_{F}^{0}}-\frac{9}{5}, \quad \eta=\frac{5 K^{0}+6 \epsilon_{F}^{0}}{18\left(\epsilon_{F}^{0}-5 \mu^{0}\right)}
$$

Here $\mu^{0}$ is the chemical potential (binding energy per nucleon at equilibrium), $K^{0}$ the compression modulus, $\epsilon_{F}^{0}$ the Fermi energy and $\beta^{0}$ the symmetry energy for infinite nuclear matter. The deduced functional parameters, eq. (2.17), correspond to $\mu^{0}=$ $-15.73 \mathrm{MeV}, K^{0}=135 \mathrm{MeV}, \epsilon_{F}^{0}=(9 \pi / 8)^{2 / 3} \hbar^{2} / 2 m r_{0}^{2}=37.35 \mathrm{MeV}\left(r_{0}=1.135\right.$ $\mathrm{fm} ; C_{0}=305 \mathrm{MeV} \cdot \mathrm{fm}^{3}$ ) and $\beta^{0}=31 \mathrm{MeV}$. The mean field potentials (see appendix A for the analytical expressions) calculated according to the procedure outlined above are used throughout this paper. It should be mentioned that the density functional described here was already succesfully applied to a description of high-precision elastic and inelastic electron scattering data on ${ }^{140} \mathrm{Ce}\left[\right.$ ref. $\left.\left.^{14}\right)\right],{ }^{118} \mathrm{Sn}\left[\right.$ ref. $^{15}$ )], ${ }^{86} \mathrm{Sr}$ [ref. ${ }^{16}$ )], ${ }^{196} \mathrm{Pt}\left[\right.$ ref. $^{17}$ )], ${ }^{89} \mathrm{Y}$ [ref. $\left.\left.{ }^{18}\right)\right]$ and also on double-magic nuclei ${ }^{40,48} \mathrm{Ca}$ and ${ }^{208} \mathrm{~Pb}$ $\left[\right.$ ref. $\left.\left.^{13}\right)\right]$.

\section{Calculation of one-phonon collective excitations}

In order to obtain the transition densities and the excitation amplitudes of low-lying collective states we use the method of ref. ${ }^{19}$ ) based on a mixed $(r, \lambda)$ representation, in which the functions related to the particle-hole channel are calculated in the coordinate 
representation including the whole continuum, while the particle-particle and hole-hole pairing correlations are treated on the basis restricted to all bound single-particle levels.

The corresponding system of QRPA-type equations reads

$$
\hat{V}=e_{q} \hat{V}_{0}+\hat{\mathcal{F}} \hat{A} \hat{V}
$$

where $e_{q}$ is the quasiparticle local charge with respect to the field $V^{(0)}\left[\right.$ ref. $\left.\left.^{11}\right)\right]$. For a long-range electric field $e_{q}=1$ as it follows from gauge invariance. Because of pairing, one has to consider particle-hole, particle-particle, and hole-hole channels, resulting in a 3 by 3 matrix formalism. The external field is written as

$$
\hat{V}_{0}=\left(\begin{array}{c}
V^{(0)} \\
0 \\
0
\end{array}\right)
$$

The elements of the effective interaction matrix,

$$
\hat{\mathcal{F}}=\left(\begin{array}{ccc}
\mathcal{F} & 0 & 0 \\
0 & -\mathcal{F}^{\xi} & 0 \\
0 & 0 & -\mathcal{F}^{\xi}
\end{array}\right)
$$

are obtained as the second functional derivatives with respect to the corresponding densities $\left(\tau, \tau^{\prime}=n, p\right)$ :

$$
\mathcal{F}^{\left(\tau \tau^{\prime}\right)}=\frac{\delta^{2} E_{\mathrm{int}}[\rho, \nu]}{\delta \rho^{\tau} \delta \rho^{\tau^{\prime}}}, \mathcal{F}^{\xi(\tau \tau)}=\frac{\delta^{2} E_{\mathrm{int}}[\rho, \nu]}{\delta \nu^{\tau} \delta \nu^{\tau}}
$$

The particle-hole interaction $\mathcal{F}^{\left(\tau \tau^{\prime}\right)}$ is described in detail in appendix B.

The components $A_{i k}$ of the propagator matrix $\hat{A}$ are obtained from the integration of various pair products of normal, $G(1,2)=<N|T \Psi(1) \Psi(2)+| N\rangle$, and anomalous, 
$F^{(1)}(1,2)=<N-2|T \Psi(1) \Psi(2)| N>, F^{(2)}(1,2)=<N+2\left|T \Psi^{+}(1) \Psi^{+}(2)\right| N>$, Green's functions over the energy variable. Using the notations adopted in refs. ${ }^{11,19}$ ), the matrix $\hat{A}$ is written as

$$
\hat{A}=\left(\begin{array}{ccc}
\mathcal{L} & \mathcal{M}_{1} & \mathcal{M}_{2} \\
\mathcal{O} & \mathcal{N}_{1} & \mathcal{N}_{2} \\
\tilde{\mathcal{O}} & \tilde{\mathcal{N}}_{1} & \tilde{\mathcal{N}}_{2}
\end{array}\right)
$$

The quantity $\hat{V}$ of eq. (3.1) is the effective field in the system resulting from the action of a "bare" external field $\hat{V}_{0}$. When the frequency $\omega$ of the external field approaches the collective excitation energy $\omega_{s}$ the system undergoes a resonance behaviour and, accordingly, $\hat{V}$ has a simple pole. The phonon excitation amplitude $\hat{g}_{s}$ is defined as the residue of the effective field at $\omega=\omega_{s}$. It is obtained as a solution of the homogeneous equation associated with eq. (3.1):

$$
\hat{g}_{s}=\hat{\mathcal{F}} \hat{A}\left(\omega_{s}\right) \hat{g}_{s}
$$

A graphical representation of this equation is shown in fig. 1. The normalisation condition for $\hat{g}_{s}$ reads as ${ }^{11}$ )

$$
\left(\hat{g}_{s}^{+} \frac{d \hat{A}}{d \omega} \hat{g}_{s}\right)_{\omega=\omega_{s}}=-1
$$

where an integration over all intermediate coordinates is implied.

For a system with pairing correlations it is convenient to deal with the conjugate equation for the transition density matrix $\hat{\rho}_{s}^{\mathrm{tr}}$, defined as

$$
\hat{\rho}_{s}^{\mathrm{tr}}=\hat{A} \hat{g}_{s}
$$


then from eq. (3.6) one gets

$$
\hat{\rho}_{s}^{\operatorname{tr}}=\hat{A}\left(\omega_{s}\right) \hat{\mathcal{F}} \hat{\rho}_{s}^{\text {tr }}
$$

This equation incorporates both the normal and anomalous transition densities

$$
\begin{aligned}
& \rho_{s}^{\operatorname{tr}(0)}\left(\vec{r}, \vec{r}^{\prime}\right)=\int \frac{\mathrm{d} \varepsilon}{2 \pi i} \delta G\left(\vec{r}, \vec{r}^{\prime} ; \varepsilon, \omega_{s}\right), \\
& \rho_{s}^{\operatorname{tr}(1,2)}\left(\vec{r}, \vec{r}^{\prime}\right)=\int \frac{\mathrm{d} \varepsilon}{2 \pi i} \delta F^{(1,2)}\left(\vec{r}, \vec{r}^{\prime} ; \varepsilon, \omega_{s}\right),
\end{aligned}
$$

where $\delta G$ and $\delta F^{(1,2)}$ are the changes of normal and anomalous Green's functions respectively due to appearing of the one-phonon excitation $s$ in the system. In the diagonal pairing approximation, $\Delta_{\lambda \lambda^{\prime}}=\Delta_{\lambda} \delta_{\lambda \lambda^{\prime}}$, and using the relations ${ }^{20}$ ) between $\rho^{\operatorname{tr}(0)}$ and $\rho^{\operatorname{tr}( \pm)}=\left(\rho^{\operatorname{tr}(1)} \pm \rho^{\operatorname{tr}(2)}\right) / 2$, one obtains a considerable simplification of the set of equations (3.1). The computational scheme is described in detail in ref. ${ }^{19}$ ). Neglecting velocity-dependent forces the phonon amplitudes $g_{s}$, and the transition densities $\rho_{s}^{\text {tr }}$, reduce to one-point functions and the transition matrix element from the ground state to the excited state $s$ is given by

$$
M_{s}=e_{q} \int \hat{V}_{0}(\vec{r}) \hat{\rho}_{s}^{\operatorname{tr}}(\vec{r}) \mathrm{d} \vec{r}
$$

\section{Equation for the transition density of two-phonon states}

In this section we shall concentrate on the main point of the present paper - the deduction and analysis of equations which allow to calculate self-consistently transition densities to two-phonon states. Since the low-energy collective modes (phonons) are assumed to be adiabatic with respect to single-particle motion, one can treat their influence on various nuclear properties, in particular the effect of a given phonon on 
the excitation amplitude of another one, by means of an external field formalism. Then matrix element $M_{s_{1} s_{2}}$ of a transition from the ground state to the excited state with two phonons $s_{1}$ and $s_{2}$ is the functional derivative $\delta_{s_{1}} M_{s_{2}}$ of the one-phonon matrix element $M_{s_{2}}$, eq. (3.11), with respect to the phonon $s_{1}$. If we introduce the quantity

$$
\delta_{s_{1}} \hat{\rho}_{s_{2}}^{\operatorname{tr}}=\left(\rho_{12}^{\operatorname{tr}(0)}, \rho_{12}^{\operatorname{tr}(1)}, \rho_{12}^{\operatorname{tr}(2)}\right) \equiv \hat{\rho}_{12}^{\operatorname{tr}}
$$

the corresponding matrix element is

$$
M_{s_{1} s_{2}}=e_{q} \int \hat{V}_{0}(\vec{r}) \hat{\rho}_{12}^{\mathrm{tr}}(\vec{r}) \mathrm{d} \vec{r}
$$

with $\hat{\rho}_{12}^{\mathrm{tr}}$ being the transition density to the two-phonon state we are looking for. The relevant equation for $\hat{\rho}_{12}^{\mathrm{tr}}$ is obtained from the variation of the homogeneous equation (3.9) for the $s_{2}$ phonon in the field of the $s_{1}$ phonon. This results in

$$
\hat{\rho}_{12}^{\mathrm{tr}}=\frac{1}{2}\left(\hat{T}_{12}+\hat{A} \hat{W} \hat{\rho}_{1}^{\mathrm{tr}} \hat{\rho}_{2}^{\mathrm{tr}}\right)+\hat{A} \hat{\mathcal{F}} \hat{\rho}_{12}^{\mathrm{tr}}
$$

where

$$
\hat{T}_{12}=\hat{G} \hat{g}_{1} \hat{G} \hat{g}_{2}+\hat{G} \hat{g}_{2} \hat{G} \hat{g}_{1}
$$

and

$$
\hat{W}=\frac{\delta \hat{\mathcal{F}}}{\delta \hat{\rho}}
$$

According to the definition of eqs. (3.4) the effective three-body interaction $W$ entering eq. (4.3) is the third functional derivative of a density functional. It is described in detail in appendix $\mathrm{C}$. 
On the other hand, applying the variation to eq. (3.8), the transition density $\hat{\rho}_{12}^{\mathrm{tr}}$ to the two-phonon state can be written as

$$
\hat{\rho}_{12}^{\mathrm{tr}}=\frac{1}{2} \hat{T}_{12}+\hat{A} \hat{g}_{12}
$$

introducing a creation amplitude $\hat{g}_{12}$ for this two-phonon state. This quantity satisfies an equation which in turn can be obtained from the variation being applied to eq. (3.6), which results in

$$
\hat{g}_{12}=\frac{1}{2}\left(\hat{\mathcal{F}} \hat{T}_{12}+\hat{W} \hat{\rho}_{1}^{\text {tr }} \hat{\rho}_{2}^{\text {tr }}\right)+\hat{\mathcal{F}} \hat{A} \hat{g}_{12}
$$

This equation is illustrated grafically in fig. 2 together with diagrams describing the matrix element, eq. (4.2), for excitation of two-phonon state in the external field $V_{0}$. The contributions to the mean fields and to the effective interactions $\mathcal{F}$ and $W$, derived self-consistently from our energy density functional to the corresponding order in the interaction vertices, are pictured in fig. 3 .

It should be stressed that eqs. (4.1)-(4.7) correspond to effects of second order in the one-phonon amplitudes $g_{s}$. Within a self-consistent harmonic approximation this yields transition densities and creation amplitudes of two-phonon state located at the energy of $\omega_{12}=\omega_{1}+\omega_{2}$. To demonstrate self-consistency of our approach we consider the special case of the action of a displacement operator $\propto \frac{\partial}{\partial r_{\alpha}}$ on the variation of our density functional (omitting here the dependence on the anomalous density $\nu$ for simplicity). To the first order in the shift we have

$$
\frac{\partial}{\partial r_{\alpha}} \frac{\delta E_{\mathrm{int}}}{\delta \rho_{i}}=\sum_{k} \frac{\delta^{2} E_{\mathrm{int}}}{\delta \rho_{i} \delta \rho_{k}} \frac{\partial \rho_{k}}{\partial r_{\alpha}} \equiv \sum_{k} \mathcal{F}^{i k} \frac{\partial \rho_{k}}{\partial r_{\alpha}}
$$


Using the identity

$$
\begin{aligned}
\frac{\partial \rho_{k}(\vec{r})}{\partial r_{\alpha}} & =\int \frac{\mathrm{d} \varepsilon}{2 \pi i} \mathrm{~d} \vec{r}^{\prime} G^{k}\left(\vec{r}, \vec{r}^{\prime} ; \varepsilon\right) \frac{\partial U^{k}\left(\vec{r}^{\prime}\right)}{\partial r_{\alpha}^{\prime}} G^{k}\left(\vec{r}^{\prime}, \vec{r} ; \varepsilon\right) \\
& \equiv \int \mathrm{d} \vec{r}^{\prime} A^{k}\left(\vec{r}, \vec{r}^{\prime} ; \omega=0\right) \frac{\partial U^{k}\left(\vec{r}^{\prime}\right)}{\partial r_{\alpha}^{\prime}}
\end{aligned}
$$

and the definitions of the mean fields [eq. (2.16)] and effective interactions [eq. (3.4)], one gets

$$
\frac{\partial U^{i}(\vec{r})}{\partial r_{\alpha}}=\sum_{k} \int \mathrm{d} \vec{r}^{\prime} \mathrm{d} \vec{r}^{\prime \prime} \mathcal{F}^{i k}\left(\vec{r}, \vec{r}^{\prime}\right) A^{k}\left(\vec{r}^{\prime}, \vec{r}^{\prime \prime} ; \omega=0\right) \frac{\partial U^{k}\left(\vec{r}^{\prime \prime}\right)}{\partial r_{\alpha}^{\prime \prime}}
$$

This equation is equivalent to eq. (3.6) in the limit of a zero-energy dipole phonon (isoscalar spurious $1^{-}$) state, the transition density and transition potential are proportional to $\partial \rho / \partial r$ and $\partial U / \partial r$ respectively as one has to expect for systems with spontaneously broken translational symmetry ${ }^{21}$ ).

In a similar way to the second order in the displacement one obtains

$$
\begin{aligned}
\frac{\partial^{2} U^{i}}{\partial r_{\alpha} \partial r_{\beta}} & =\sum_{k l} \frac{\delta^{3} E_{\mathrm{int}}}{\delta \rho_{i} \delta \rho_{k} \delta \rho_{l}} \frac{\partial \rho_{k}}{\partial r_{\alpha}} \frac{\partial \rho_{l}}{\partial r_{\beta}}+\sum_{k} \frac{\delta^{2} E_{\mathrm{int}}}{\delta \rho_{i} \delta \rho_{k}} \frac{\partial^{2} \rho_{k}}{\partial r_{\alpha} \partial r_{\beta}} \\
& \equiv \sum_{k l} W^{i k l} \frac{\partial \rho_{k}}{\partial r_{\alpha}} \frac{\partial \rho_{l}}{\partial r_{\beta}}+\sum_{k} \mathcal{F}^{i k} \frac{\partial^{2} \rho_{k}}{\partial r_{\alpha} \partial r_{\beta}}
\end{aligned}
$$

The identity containing the derivatives of the mean field and the densities in this order reads

$$
\begin{aligned}
\frac{\partial^{2} \rho}{\partial r_{\alpha} \partial r_{\beta}} & =\left[G \frac{\partial U}{\partial r_{\alpha}} G \frac{\partial U}{\partial r_{\beta}} G+G \frac{\partial U}{\partial r_{\beta}} G \frac{\partial U}{\partial r_{\alpha}} G\right]+G \frac{\partial^{2} U}{\partial r_{\alpha} \partial r_{\beta}} G \\
& \equiv T_{12(\alpha \beta)}(\omega=0)+A(\omega=0) \frac{\partial^{2} U}{\partial r_{\alpha} \partial r_{\beta}} .
\end{aligned}
$$

(Remember that for simplicity we often do not show integrations over space and energy variables). We thus have shown that the solutions of eqs. (4.3) and (4.7), provided 
the effective three-body interaction is defined self-consistently by eq. (4.5), give the transition density $\rho_{12(\alpha \beta)}^{\mathrm{tr}, k}=\frac{1}{2} \frac{\partial^{2} \rho^{k}}{\partial r_{\alpha} \partial r_{\beta}}$ and the transition potential $g_{12(\alpha \beta)}^{k}=\frac{1}{2} \frac{\partial^{2} U^{k}}{\partial r_{\alpha} \partial r_{\beta}}$ to the "double-dipole spurious state", the factors $\frac{1}{2}$ appear as usual from the expansion in second order. It should be stressed that all relations considered in these examples are strictly valid if one uses not only self-consistently derived effective interactions but also complete particle-hole basis including continuum.

To discuss the procedure to obtain the transition density to two-phonon state, let us first rewrite the equation for $\hat{T}_{12}$ for a case without pairing, having the normal Green's function $G$ only. Using the relation $\delta_{1} G=G g_{1} G$, we get

$$
\begin{aligned}
T_{12}(\vec{r}) & =\int \mathrm{d} \vec{r}_{1} \mathrm{~d} \vec{r}_{2} \frac{\mathrm{d} \varepsilon}{2 \pi i} G\left(\vec{r}, \vec{r}_{1} ; \varepsilon\right) g_{1}\left(\vec{r}_{1}\right) \times \\
& \times G\left(\vec{r}_{1}, \vec{r}_{2} ; \varepsilon-\omega_{1}\right) g_{2}\left(\vec{r}_{2}\right) G\left(\vec{r}_{2}, \vec{r} ; \varepsilon-\omega_{1}-\omega_{2}\right) .
\end{aligned}
$$

An expression suitable for numerical calculations can be obtained by integrating this equation over $\varepsilon$ and by separating the angular variables. For the external fields of electric-type symmetry we have

$$
T_{12}^{J}(\vec{r})=(-1)^{M+1}\left(\begin{array}{ccc}
L_{1} & L_{2} & J \\
M_{1} & M_{2} & -M
\end{array}\right) Y_{J M}(\vec{n}) t_{L_{1} L_{2}}^{J}(r)
$$

where conventional notations for the $J$ th spherical function and the $3 j$ symbol are used. The radial part of $T_{12}^{J}$ is given by 


$$
\begin{aligned}
& t_{L_{1} L_{2}}^{J}(r)=\int r_{1}^{2} \mathrm{~d} r_{1} r_{2}^{2} \mathrm{~d} r_{2} g_{L_{1}}\left(r_{1}\right) g_{L_{2}}\left(r_{2}\right) \sum_{\lambda, l^{\prime} j^{\prime} l^{\prime \prime} j^{\prime \prime}} n_{\lambda} \times \\
& \times\left[\left(l j\left\|Y_{J}\right\| l^{\prime \prime} j^{\prime \prime}\right)\left(l^{\prime \prime} j^{\prime \prime}\left\|Y_{L_{2}}\right\| l^{\prime} j^{\prime}\right)\left(l^{\prime} j^{\prime}\left\|Y_{L_{1}}\right\| l j\right)\left\{\begin{array}{ccc}
L_{2} & J & L_{1} \\
j & j^{\prime} & j^{\prime \prime}
\end{array}\right\} \times\right. \\
& \times R_{n l j}\left(r_{1}\right) R_{n l j}(r) G_{l^{\prime} j^{\prime}}\left(r_{1}, r_{2} ; \varepsilon_{\lambda}-\omega_{1}\right) G_{l^{\prime \prime} j^{\prime \prime}}\left(r_{2}, r ; \varepsilon_{\lambda}-\omega_{1}-\omega_{2}\right)+ \\
& +\left(l j\left\|Y_{L_{1}}\right\| l^{\prime \prime} j^{\prime \prime}\right)\left(l^{\prime \prime} j^{\prime \prime}\left\|Y_{J}\right\| l^{\prime} j^{\prime}\right)\left(l^{\prime} j^{\prime}\left\|Y_{L_{2}}\right\| l j\right)\left\{\begin{array}{ccc}
J & L_{1} & L_{2} \\
j & j^{\prime} & j^{\prime \prime}
\end{array}\right\} \times \\
& \times R_{n l j}\left(r_{1}\right) R_{n l j}\left(r_{2}\right) G_{l^{\prime} j^{\prime}}\left(r_{2}, r ; \varepsilon_{\lambda}-\omega_{2}\right) G_{l^{\prime \prime} j^{\prime \prime}}\left(r, r_{1} ; \varepsilon_{\lambda}+\omega_{1}\right)+ \\
& +\left(l j\left\|Y_{L_{2}}\right\| l^{\prime \prime} j^{\prime \prime}\right)\left(l^{\prime \prime} j^{\prime \prime}\left\|Y_{L_{1}}\right\| l^{\prime} j^{\prime}\right)\left(l^{\prime} j^{\prime}\left\|Y_{J}\right\| l j\right)\left\{\begin{array}{ccc}
L_{1} & L_{2} & J \\
j & j^{\prime} & j^{\prime \prime}
\end{array}\right\} \times \\
& \left.\times R_{n l j}\left(r_{2}\right) R_{n l j}(r) G_{l^{\prime} j^{\prime}}\left(r, r_{1} ; \varepsilon_{\lambda}+\omega_{1}+\omega_{2}\right) G_{l^{\prime \prime} j^{\prime \prime}}\left(r_{1}, r_{2} ; \varepsilon_{\lambda}+\omega_{2}\right)\right] \text {. }
\end{aligned}
$$

Using this expression, it can be easily shown that in the case of $L_{1}=L_{2}=1$ and $\omega=0$ there are two possible two-phonon spurious states with $J=0$ and $J=2$, which correspond to an expansion of second order displacement in irreducible spherical tensors, the radial transition densities being $\rho_{11}^{\operatorname{tr}, J=0}=\frac{1}{2}\left(\frac{\partial^{2} \rho}{\partial r^{2}}+\frac{2}{r} \frac{\partial \rho}{\partial r}\right)$ and $\rho_{11}^{\operatorname{tr}, J=2}=$ $\frac{1}{2}\left(\frac{\partial^{2} \rho}{\partial r^{2}}-\frac{1}{r} \frac{\partial \rho}{\partial r}\right)$ respectively.

The situation becomes more complicated for systems with pairing owing to the existence of the anomalous Green's functions, which transform a particle into a hole and vice versa, and also the anomalous phonon excitation amplitudes. However, in sufficiently "stiff" nuclei, far from the region of the phase transition to the deformed state, the normal particle-hole component of the effective field dominates and this allows us to a certain extent to simplify the analysis [the calculations of ref. ${ }^{19}$ ) showed that the position of the first collective quadrupole excitation may serve as the practical criterion for "stiffness" of the nucleus]. By selecting the corresponding Feynman diagrams and omitting the dependence on the space coordinates and spin-isospin variables we obtain, 
as in the case of eq. (4.13),

$$
\begin{aligned}
T_{12} & =\int \frac{\mathrm{d} \varepsilon}{2 \pi i} g_{1} g_{2}\left[G(\varepsilon) G\left(\varepsilon-\omega_{1}\right) G\left(\varepsilon-\omega_{1}-\omega_{2}\right)-\right. \\
& -G(\varepsilon) F^{(1)}\left(\varepsilon-\omega_{1}\right) F^{(2)}\left(\varepsilon-\omega_{1}-\omega_{2}\right)-F^{(1)}(\varepsilon) F^{(2)}\left(\varepsilon-\omega_{1}\right) G\left(\varepsilon-\omega_{1}-\omega_{2}\right)- \\
& \left.-F^{(1)}(\varepsilon) G^{T}\left(\varepsilon-\omega_{1}\right) F^{(2)}\left(\varepsilon-\omega_{1}-\omega_{2}\right)\right] .
\end{aligned}
$$

Here, $G^{T}$ is the Green's function obtained under time reversal, $G_{\sigma \sigma^{\prime}}^{T}(\varepsilon)=G_{\sigma^{\prime} \sigma}(-\varepsilon)$ ( $\alpha$ and $\beta$ are the spin variables). Taking into account the fact that $F^{(1)} \equiv F^{(2)}$ in the diagonal pairing approximation and using the expansions of ref. ${ }^{11}$ ) for the Green's functions in the $\lambda$ representation, we have

$$
\begin{aligned}
& G\left(\vec{r}, \vec{r}^{\prime} ; \varepsilon\right)=\sum_{\lambda} \varphi_{\lambda}^{+}(\vec{r}) \varphi_{\lambda}\left(\vec{r}^{\prime}\right)\left\{\frac{u_{\lambda}^{2}}{\varepsilon-E_{\lambda}+i \gamma}+\frac{v_{\lambda}^{2}}{\varepsilon+E_{\lambda}-i \gamma}\right\} \\
& F\left(\vec{r}, \vec{r}^{\prime} ; \varepsilon\right)=-\sum_{\lambda} \varphi_{\lambda}^{+}(\vec{r}) \varphi_{\lambda}\left(\vec{r}^{\prime}\right) \frac{\Delta_{\lambda}}{2 E_{\lambda}}\left\{\frac{1}{\varepsilon-E_{\lambda}+i \gamma}+\frac{1}{\varepsilon+E_{\lambda}-i \gamma}\right\}
\end{aligned}
$$

where $E_{\lambda}=\sqrt{\varepsilon_{\lambda}^{2}+\Delta_{\lambda}^{2}}, v_{\lambda}^{2}=\left(E_{\lambda}-\varepsilon_{\lambda}\right) / 2 E_{\lambda}$, and $v_{\lambda}^{2}+u_{\lambda}^{2}=1\left(\varepsilon_{\lambda}\right.$ is measured from the chemical potential $\mu$ ). In order to give a more compact form for the result of the integration over $\varepsilon$, we introduce the "generalized" Green's function $\hat{\mathcal{G}}(\varepsilon)$ and occupation factors $\hat{\zeta}_{\lambda}$ defined by

$$
\hat{\mathcal{G}}(\varepsilon)=\left\{\begin{array}{c}
G(\varepsilon) \\
F(\varepsilon) \\
G^{T}(\varepsilon)
\end{array}\right\} ; \quad \hat{\zeta}_{\lambda}=\left\{\begin{array}{c}
v_{\lambda}^{2} \\
\Delta_{\lambda} / 2 E_{\lambda} \\
-u_{\lambda}^{2}
\end{array}\right\}
$$

Then each of the three terms in eq. (4.16) can be written as 


$$
\begin{aligned}
T_{12}^{i j k}(\vec{r}) & =\int r_{1}^{2} \mathrm{~d} r_{1} r_{2}^{2} \mathrm{~d} r_{2} g L_{1}\left(r_{1}\right) g_{L_{2}}\left(r_{2}\right) \times \\
& \times \sum_{\lambda_{1} \lambda_{2} \lambda_{3}} \varphi_{\lambda_{1}}^{+}(\vec{r}) \varphi \lambda_{3}(\vec{r}) \varphi_{\lambda_{2}}^{+}\left(\vec{r}_{1}\right) \varphi_{\lambda_{1}}\left(\vec{r}_{1}\right) \varphi_{\lambda_{3}}^{+}\left(\vec{r}_{2}\right) \varphi_{\lambda_{2}}\left(\vec{r}_{2}\right) \times \\
& \times\left[\zeta_{\lambda_{1}}^{(i)} \mathcal{G}_{\lambda_{2}}^{(j)}\left(-E_{\lambda_{1}}-\omega_{1}\right) \mathcal{G}_{\lambda_{3}}^{(k)}\left(-E_{\lambda_{1}}-\omega_{1}-\omega_{2}\right)+\right. \\
& +\zeta_{\lambda_{2}}^{(j)} \mathcal{G}_{\lambda_{1}}^{(k)}\left(-E_{\lambda_{2}}+\omega_{1}\right) \mathcal{G}_{\lambda_{3}}^{(i)}\left(-E_{\lambda_{2}}-\omega_{2}\right)+ \\
& \left.+\zeta_{\lambda_{3}}^{(k)} \mathcal{G}_{\lambda_{1}}^{(i)}\left(-E_{\lambda_{3}}+\omega_{1}+\omega_{2}\right) \mathcal{G}_{\lambda_{2}}^{(j)}\left(-E_{\lambda_{3}}-\omega_{2}\right)\right]
\end{aligned}
$$

The indices $i, j, k$ run from 1 to 3 and label the definite components of the vectors $\hat{\mathcal{G}}$ and $\hat{\zeta}_{\lambda}$. The normal Green's function $G$ is composed of two parts; the first part does not manifestly contain pairing and can be calculated according to the scheme of refs. ${ }^{3,4}$ ) as a combination of two linearly independent solutions of the Schrödinger equation with suitable boundary conditions. The second part of $G$, and also the functions $F$ and $G^{T}$, are represented as a sum over the bound states forming a valence space. The advantage of this scheme is the possibility of taking into account all the particle-hole transitions completely including the continuum.

\section{Calculations and discussion of results}

\section{5a. Calculations}

We have applied the approach outlined above to the microscopic self-consistent description of some low-lying collective one- and two-phonon states in the ${ }^{96} \mathrm{Zr}$ nucleus for which new experimental data now are available ${ }^{1}$ ). As input, only the basic set of density functional parameters given by eq. (2.17) was utilized.

We determine the $B(E L \uparrow)$ and $\beta_{L}$ values as usual by

$$
B(E L \uparrow)=(2 L+1)\left(\int \rho_{p}^{\operatorname{tr}} r^{L+2} \mathrm{~d} r\right)^{2}=e^{2} \beta_{L}^{2} \frac{9 Z^{2}}{16 \pi^{2}} R^{2 L}
$$

For comparison with the $B(E \lambda \downarrow)$ values measured by $\gamma$-deexcitations the $B(E \lambda \uparrow)$ 
values given in eq. (5.1) are divided by $(2 L+1)$ and expressed in the Weisskopf units:

$$
1 \text { W.u. }=\frac{e^{2}}{4 \pi}\left(\frac{3}{L+3}\right)^{2} R^{2 L}
$$

where $R=1.2 A^{1 / 3} \mathrm{fm}$.

From eq. (3.6) the first $3^{-}$state was obtained at $E\left(3_{1}^{-}\right)=1.74 \mathrm{MeV}$ with $B(E 3 \uparrow)=$ $1.51 \cdot 10^{5} e^{2} \mathrm{fm}^{6}$ (this corresponds to a $B(E 3 \downarrow)$ value of 39.5 W.u. or to a deformation parameter $\left.\beta_{3} \approx 0.246\right)$. The first one-phonon $6^{+}$excitation was obtained at $E\left(6_{1}^{+}\right)$ $=3.51 \mathrm{MeV}$ with $B(E 6 \uparrow)=1.08 \cdot 10^{8} e^{2} \mathrm{fm}^{12}$ (or $1.24 \mathrm{~W}$.u. and $\beta_{6} \approx 0.04$ ). This excitation is built mainly on the neutron particle-hole configuration $\left(\nu 1 g_{7 / 2}, \nu 2 d_{5 / 2}^{-1}\right)$. No other $6^{+}$one-phonon states were found below $4.5 \mathrm{MeV}$.

Both $3_{1}^{-}$and $6_{1}^{+}$state occured to be slightly sensitive to the variation of the pairing strength constant $f^{\xi}$. Taking $f^{\xi}=-0.38$ instead of -0.33 as in the basic set, we get for the $3_{1}^{-}$level $E\left(3_{1}^{-}\right)=2.16 \mathrm{MeV}$ and $B(E 3 \downarrow)=34.7$ W.u. and, for the $6_{1}^{+}$state, $E\left(6_{1}^{+}\right)$ $=3.31 \mathrm{MeV}$ and $B(E 6 \downarrow)=1.13 \mathrm{~W}$. u. On the other hand, pairing correlations produce rather strong effect on the $2_{1}^{+}$state. For the basic set we have $E\left(2_{1}^{+}\right)=1.34 \mathrm{MeV}$ and $B(E 2 \downarrow)=0.85$ W.u. while at $f^{\xi}=-0.38, E\left(2_{1}^{+}\right)=1.51 \mathrm{MeV}$ and $B(E 2 \downarrow)=3.98$ W.u. This sensitivity is mainly related with changes of the occupation numbers and of the energies of positive parity $\left(2 d_{5 / 2}, 3 s_{1 / 2}, 2 d_{3 / 2}\right.$ and $\left.1 g_{7 / 2}\right)$ neutron quasiparticle levels near the Fermi surface. Regarding pairing, the situation in ${ }^{96} \mathrm{Zr}$ occurs to be close to the point of phase transition to the superfluid state (a critical value of the pairing interaction constant is $f_{c r}^{\xi}=-0.31$ ). We conclude that in such a situation the concept of volume pairing with only one strength constant of local interaction (2.14) is oversimplified, for a proper description of low-lying quadrupole exitations one might need a more sophisticated model with a density-dependent pairing term $\varepsilon_{\text {pair }}$. 
It is interesting to study the isospin character of the transitions to the excited collective states under consideration. For that purpose one often uses the ratio of the neutron to proton multipole matrix elements, i.e. $M_{n}^{l} / M_{p}^{l}$, where

$$
M_{n, p}^{l}=\int \mathrm{d} r r^{l+2} \rho_{n, p}^{\mathrm{tr}}
$$

For the $3_{1}^{-}$state our calculation give $M_{n} / M_{p}=1.39$, almost the expected ratio of 1.40 ( $=N / Z$ for ${ }^{96} \mathrm{Zr}$ ). For the first $2^{+}$state we obtained $M_{n} / M_{p}=2.59$ and 1.84 at $f^{\xi}=-0.33$ and -0.38 , respectively, showing again the important role played by pairing correlations in the microscopic description of the $2_{1}^{+}$state properties in ${ }^{96} \mathrm{Zr}$.

Now we concentrate on the most important point of the present paper, which is to investigate the mixing of the two-phonon configuration $\left[3_{1}^{-} \otimes 3_{1}^{-}\right]$, formed by the strongly collective octupole vibration with a large parameter of dynamical deformation $\beta_{3}$, and the one-phonon $6_{1}^{+}$excitation of rather weak collectivity and a low value of $\beta_{6}$. Since $\beta_{3}^{2}$ occurs to be comparable with $\beta_{6}$, one- and two-phonon $6^{+}$configurations compete with each other, and in the case of the nearly magic nucleus ${ }^{96} \mathrm{Zr}$ the calculated $B(E 6)$ value for the two-phonon configuration even dominates. The two-phonon configuration gives rise to a second $6^{+}$state which is absent in the one-phonon self-consistent QRPA scheme. For such a study one may consider ${ }^{96} \mathrm{Zr}$ as a good object because both $3^{-}$and $6^{+}$states are characterized by a rather weak anharmonicity and (in contrast to the $2^{+}$ state) pairing plays no significant role, thus our calculations are fully self-consistent.

In the present case the two configurations are almost degenerate since their unperturbed energies almost exactly satisfy the relation $2 E\left(3_{1}^{-}\right) \approx E\left(6_{1}^{+}, 1-\right.$ phonon $)$. This 
results in two states,

$$
\begin{aligned}
& \left|6^{+}\right\rangle_{+}=\frac{1}{\sqrt{2}}\left(\mid 6^{+}, 1-\text { phonon }>+\mid 6^{+}, 2-\text { phonon }>\right), \\
& \left|6^{+}\right\rangle_{-}=\frac{1}{\sqrt{2}}\left(-\mid 6^{+}, 1-\text { phonon }>+\mid 6^{+}, 2-\text { phonon }>\right) .
\end{aligned}
$$

The splitting of these two states, $\Delta E=2\left|V_{1 p h-2 p h}\right|$, is determined by the matrix element

$$
V_{1 p h-2 p h}=\sum_{i=n, p} \int \mathrm{d} \vec{r} g_{1 p h}^{(i)}(\vec{r}) \rho_{2 p h}^{\operatorname{tr}(i)}(\vec{r}),
$$

which is calculated by the integration of the product of the double-octupole two-phonon $6^{+}$transition density $\rho_{2 p h}^{\mathrm{tr}}$, being obtained from eq. (4.3), and the one-phonon $6^{+}$ amplitude $g_{1 p h}$, the latter being solution of eq. (3.6). A graphical representation of $V_{1 p h-2 p h}$ is shown in fig. 4 where the two diagrams correspond to the definition of two-phonon transition density given by the two terms in eq. (4.6). It was found that the proton component of the first diagram makes the main contribution $(\approx-130 \mathrm{keV})$ to $V_{1 p h-2 p h}$. As result we get $\Delta E=2\left|V_{1 p h-2 p h}\right| \approx 300 \mathrm{keV}$. In our calculations we did not take into account the shift of the energy of the $6^{+}$state, the member of the multiplet $\left[3_{1}^{-} \otimes 3_{1}^{-}\right]$, due to the effects of anharmonicity which come, in particular, from an interaction between double-octupole excitations. A microscopic evaluation of the splitting of this multiplet is a rather complicated problem and will be considered in a later paper.

The calculated ground-state density and the transition densities of one-phonon, two-phonon and "mixed" excited states are shown in figs. 5a-5e. It is seen from fig. $5 \mathrm{c}$ and $5 \mathrm{~d}$ that $\rho_{1-p h o n o n}^{\mathrm{tr}}$ and $\rho_{2-p h o n o n}^{\mathrm{tr}}$ are similar in shapes and comparable in amplitudes, but their maxima and minima are shifted relative to each other. It is well known $^{8,19}$ ) that the maxima of the transition densities of low-lying collective states 
calculated in a self-consistent one-phonon approximation (QRPA) are located on the surface of nucleus. Their shapes closely resemble $\frac{\mathrm{d} \rho}{\mathrm{d} r}$, where $\rho$ is the ground state density of nucleus (fig. 5a). As it was discussed in the foregoing section, a self-consistent solution of eq. (4.3) for the transition density to a double-dipole two-phonon state (second order displacement of the system as a whole) gives the result $\rho_{11(i k)}^{\mathrm{tr}} \sim \frac{\mathrm{d}^{2} \rho}{\mathrm{d} r_{i} \mathrm{~d} r_{k}}$. Of course, the fine structure of $\rho_{33}^{\mathrm{tr}}$ in the interior of nucleus differs from that of $\rho_{11}^{\mathrm{tr}}$ due to quantum fluctuations, the classical behaviour (the dashed lines in fig. 5d) is observed here only for the very tail of the transition formfactor beyond $r=6 \mathrm{fm}$.

Since the calculated $6^{+}$transition densities $\rho_{1-p h o n o n}^{\mathrm{tr}}$ and $\rho_{2-p h o n o n}^{\operatorname{tr}}$ are comparable, the resulting mixed distributions $\rho_{+}^{\text {tr }}$ and $\rho_{-}^{\text {tr }}$ (fig. 5e) occur to be sharply different in the amplitudes, their surface components produce the main contributions to the $B(E L)$ values. Hence it is not surprising that the corresponding excitation probabilities differ by a factor of about 10 :

$$
\begin{aligned}
& B(E L)_{+}=4.16 \cdot 10^{8} e^{2} \mathrm{fm}^{12}(4.78 \text { W.u. }) \\
& B(E L)_{-}=0.33 \cdot 10^{8} e^{2} \mathrm{fm}^{12} \text { (0.37 W.u.). }
\end{aligned}
$$

\section{5b. Comparison with other calculations and with experimental results}

The results of our calculation for the ground state, the $2_{1}^{+}, 3_{1}^{-}$and the $6_{1}^{+}$onephonon states and the $6^{+}$two-phonon state and the mixing of the $6^{+}(1$-phonon $)$ and $6^{+}(2$-phonon $)$ are summarized in table 1 and partly displayed in the figs. 5a-e. We compare the QRPA results with recent calculations of Horen et al. ${ }^{22}$ ) and Rosso et $a l .{ }^{2}$ ) and with the experimental observations of Hofer et $a .^{1}$ ), based primarily on the analysis of (d,d') experiments.

In contrast to the $B(E \lambda \downarrow)=M_{p}^{2}(\lambda)$ determinations from $\gamma$-deexcitations of Molnar et al. $^{23}$ ), Ohm et al. ${ }^{24}$ ) and Mach et al. ${ }^{25}$ ) the absolute values of the (d,d') 
differential cross sections are determined mainly by the respective isoscalar transition probabilities

$$
B(I \lambda \downarrow)=\left|M_{p}(\lambda)+M_{n}(\lambda)\right|^{2} \frac{Z^{2}}{A^{2}}=B(E \lambda \downarrow)\left[1+\frac{M_{n}(\lambda)}{M_{p}(\lambda)}\right]^{2} \frac{Z^{2}}{A^{2}}
$$

They are identical to the $B(E \lambda \downarrow)$, if $\frac{M_{n}(\lambda)}{M_{p}(\lambda)}=\frac{N}{Z}$. Thus we list in table 1 the transition moments $M_{n}(\lambda), M_{p}(\lambda)$, their ratio, the charge and the isoscalar transition probabilities $B(E \lambda \downarrow)$ and $B(I \lambda)$ in single particle units and, as an information about the radial shape of the respective form factors, the rms radii. The ground state distributions (fig. 5a) show some deviations from a Fermi-distribution below $r=3 \mathrm{fm}$ and a $0.18 \mathrm{fm}$ larger neutron than proton rms radius. For the $2_{1}^{+}$and the $3_{1}^{-}$states, the proton transition moments $M_{p}(\lambda)=10.2 \mathrm{fm}^{2}$ and $147 \mathrm{fm}^{3}$ are almost identical and $10 \%$ larger than the respective values of Horen et al. ${ }^{22}$ ) of $23.3 / \sqrt{2 I+1}=10.4 \mathrm{fm}^{2}$ and $346 / \sqrt{2 I+1}=131 \mathrm{fm}^{3}$, but $21 \%$ and $15 \%$ smaller than the respective values of Rosso et al. $\left.^{2}\right)\left(\left[833 \mathrm{fm}^{4} /(2 I+1)\right]^{1 / 2}=12.9 \mathrm{fm}^{2}\right.$ and $\left.\left[2.08 \cdot 10^{5} \mathrm{fm}^{6} /(2 I+1)\right]^{1 / 2}=172 \mathrm{fm}^{3}\right)$.

The calculated ratios of $M_{n} / M_{p}$ of 1.84 and 1.39 for the $2_{1}^{+}$and $3_{1}^{-}$states agree with those calculated by Horen et al. ${ }^{22}$ ) within an open-shell spherical RPA using a simple separable multipole force and harmonic-oscillator wave functions, if they are scaled by the respective power $\lambda$ of the neutron to proton radii of the ground state distribution $\left(\left\langle r_{n}^{2}\right\rangle /\left\langle r_{p}^{2}\right\rangle\right)^{1 / 2}=1.043 ; 1.66 \cdot(1.043)^{2}=1.81$ and $1.22 \cdot(1.043)^{3}=1.38$.

Fig. 5b displays the obtained neutron point transition density for the $3_{1}^{-}$state, which is compared with the first derivative $\mathrm{d} \rho / \mathrm{d} r$ of the ground state neutron density. For $r>4 \mathrm{fm}$ the two curves are in excellent agreement and thus very much in support of the collective character of the $3_{1}^{-}$state. Similar behaviour is observed for the proton transition density. 
For $6^{+}$states, the calculations yield two nearly degenerate states: a pure $6^{+}(1-$ phonon) state (fig. 5c shows the respective neutron transition density) and a $6^{+}(2-$ phonon) state with a transition density for direct (=one step) excitation from the ground state, shown in fig. 5d. The QRPA calculations of Rosso et al. ${ }^{2}$ ) for ${ }^{96} \mathrm{Zr}$ do not include the possibility of two phonon excitations. In fig. $5 \mathrm{~d}$ the radial shape of the microscopically calculated neutron transition density (the proton transition density has a similar behaviour) is dominated by a peak with the maximum near $r=5.8 \mathrm{fm}$; compared with the one-phonon transition form factor (fig. 5c), the peak is shifted by $0.6 \mathrm{fm}$ to larger radii. From the collective model a second derivative shape for this form factor (the dashed line in fig. 5d) is expected, which derives from the second order term of the Taylor expansion of the ground state density. At radii larger than $5 \mathrm{fm}$ the calculated microscopic neutron transition density for the 2 -phonon $6^{+}$state only weakly reflects a second derivative behaviour (characterized by its negative values).

Having in mind that many reactions are rather insensitive to the interior part below $r=5 \mathrm{fm}$, the use of a collective first derivative form factor is a reasonable choice for a phenomenological parametrisation of transition form factors as it was used in the study of Hofer et al. ${ }^{1}$ )

Due to the calculation giving an approximate degeneracy of the $6^{+}(1-$ phonon) and the $6^{+}$(2-phonon) states and a strength of the mixing matrix element $V_{12}=150 \mathrm{keV}$ between these states, one has to expect two $6^{+}$states, with a mixing angle near $\pm 45^{\circ}$, separated in energy by $\Delta E=2\left|V_{12}\right|=300 \mathrm{keV}$. Their transition densities result from constructive $\left(6_{1(+)}^{+}\right)$and destructive $\left(6_{2(-)}^{+}\right)$superposition and are shown in fig. 5e as curve 3 and 4 respectively. Their $B(E 6 \downarrow)$ values are 4.78 and 0.37 W.u., respectively. Due to the strong neutron contribution in the excitation of the $6_{1(+)}^{+}: M_{n} / M_{p}=2.6$ the resulting $B(I 6)=10.8$ W.u.. For the $6_{2}^{+}(-)$the ratio of $M_{n} / M_{p}$ is -1.72 . The 
negative neutron moment leads to an extremely small $B(I 6)$ value of 0.034 W.u.. The isoscalar transition probabilities $B(I \lambda)$ of the two $6^{+}$states now can be compared with the results of a DWBA analysis of deuteron scattering cross section data. We do not discuss proton scattering data since the cross sections are affected largely by exchange amplitudes - as discussed in ref. ${ }^{1}$ ) - and which are beyond the present discussion. Deuterons as composite, isoscalar probes are more sensitive to the external part of the transition potential. As discussed in ref. ${ }^{1}$ ), the cross sections in inelastic scattering, analysed with transition potentials of a radial dependence as given by the collective model, determine isoscalar transition moments. Due to the complex behaviour of the loosely bound deuteron projectile in scattering we do not attempt to use folded transition potentials derived from the calculated transition densities. Instead, we use collective transition potentials with a strength adjusted to obtain, within the procedure outlined in ref. ${ }^{1}$ ), the isoscalar moments determined by the microscopic calculation.

In this procedure, the calculated $B(I 6)$ value of the $6_{1(+)}^{+}$state, $10.8 \mathrm{~W} . \mathrm{u}$, happens to coincide with that obtained by Hofer et al. from a DWBA analysis of the 22 and $52 \mathrm{MeV}$ (d,d') experiments for the $6_{1}^{+}$state at $E_{x}=3.483 \mathrm{MeV}: B(I 6)=10.9$ W.u.. For the tentative assigned $6_{2}^{+}$state at $E_{x}=3.772 \mathrm{MeV}$, that is $289 \mathrm{keV}$ separated, the highest resolution $22 \mathrm{MeV}$ experiment yields $B(I 6)=0.5$ W.u., a factor of 15 larger than the value $0.034 \mathrm{~W} . u$. predicted by our microscopic calculations. However the $6^{+}$ assignment of the state at $3.772 \mathrm{MeV}$ results from the ${ }^{96} Y 8^{+} \beta^{-}$decay measurement cited in ${ }^{23}$ ) and is not strongly supported by the (d,d') data. Due to the complicated radial shape of the $62_{2(-)}^{+}$transition amplitude (fig. 5e shows the neutron component only), the collective model is not appropriate to describe details, but nevertheless experimental (if the $6^{+}$assignment is correct) and theory agree that the $6_{2}^{+}$cross section is small. The figs. $6 \mathrm{a}$ and $6 \mathrm{~b}$ show the $22 \mathrm{MeV}\left(\mathrm{d}, \mathrm{d}^{\prime}\right)$ data of the two $6^{+}$states with the 
discussed DWBA calculations from ref. ${ }^{1}$ ) as full curves. For the $2_{1}^{+}$and $3_{1}^{-}$transition the calculated $B(I 2)=5.57 \mathrm{~W}$. u. and $B(I 3)=39.2 \mathrm{~W}$.u. compare with 7 and $50 \mathrm{~W}$.u. from the (d,d') experiment of Hofer et al. $\left.{ }^{1}\right)$.

For the $3_{1}^{-}$state, our calculated $B(I 3)$ value of 39.2 W.u. practically coincides with the calculated $B(E 3 \downarrow)$ value. It is considerably smaller than the $B(E 3 \downarrow)=$ $65 \pm 10 \mathrm{~W} . \mathrm{u}$. reported recently by Mach et al. ${ }^{25}$ ), and somewhat smaller than $B(I 3)=$ 50 W.u., obtained from $\left.\left(\mathrm{d}, \mathrm{d}^{\prime}\right)^{1}\right)$. Following the discussion of Mach et al. ${ }^{25}$ ), the large experimental $B(E 3 \downarrow)$ values may be correlated with some anharmonicities they discussed in the deformation energy surface formalism, and which are beyond the QRPA model.

For the $6^{+}$states observed in (d,d') (see fig.6), the DWBA results mentioned above do not include sequential two step excitations via an intermediate $3_{1}^{-}$state. For pure sequential excitation building on the $3_{1}^{-}$excitation with the predicted $B(I 3)$ strength of 39.2 W.u. the result of a coupled channels calculations using collective form factors is shown as dashed curves in fig. $6 \mathrm{a}$ and fig. $6 \mathrm{~b}$. The calculated cross section has approximately the right order of magnitude when compared with the experimental cross section of the $6_{1}^{+}$state. The two step amplitude, however, interferes with the one step excitation amplitude and reduces or enhances cross section. In this concept the analysis of Hofer et al. ${ }^{1}$ ) obtained for the $6_{1}^{+}$state a destructive interference of a direct amplitude with a small $B(I 6)$ value of 3.1 W.u. and only an $18 \%$ contribution of sequential excitation, based however on their observed $B(I 3)$ value of $50 \mathrm{~W} . \mathrm{u}$.

The results of coupled channels calculations for the two $6^{+}$states which include the mixing of a pure two step excitations building on the $3_{1}^{-}$state with the calculated $B(I 3)=39.2$ W.u. with a one step excitation according to eq.(5.4) and with the effective $B(I 6)$ values of 10.8 W.u. and 0.034 W.u. for the $6_{1}^{+}$and the $6_{2}^{+}$states, respectively, 
are shown as dotted curves in fig. 6a and fig. 6b. The dotted curves in fig. 6a are calculated with a mixing angle of $+45^{\circ}$ as it is the result of our microscopic calculation. For the $6_{1}^{+}$state, the calculated cross section underpredicts the experimental one and the shape of the analyzing power is completely wrong. The cross section of the $62_{2}^{+}$state is overpredicted by more than a factor of 5 . The dotted curves of fig. $6 \mathrm{~b}$ show the result of the same type of calculation but with mixing angle $+135^{\circ}$. The shapes of cross sections and analyzing powers are much better described in this case but the strengths of the experimental observables again are not reproduced.

\section{Conclusions}

In the present paper we have formulated a self-consistent approach to the description of two-phonon excitations in nuclei far from the region of phase transition to the deformed state. In this case, smallness of the dynamical deformation $\alpha_{L}=\beta_{L} R / \sqrt{2 L+1}$ as compared with the nuclear radius $R$ allows to restrict calculations within the perturbation theory to second order in $\alpha / R$. One more assumption, essential for the present approach, is an adiabaticity of the collective motion with respect to the single-particle one. This allows to treat phonons as an external field for the single-particle degrees of freedom.

We have described the scheme of calculation of the self-consistent potential starting from a modified version of energy density functional where the density dependence of the volume terms was simulated by simple fractional-linear functions and the surface terms were related with density-dependent finite-range forces. This functional enables to reproduce the experimental data not only for ground state, but also for low-lying collective excitations.

The equations, which allow to calculate microscopically the transition densities of 
two-phonon states on the self-consistent quasiparticle basis, were obtained. The calculation scheme was realized for the ${ }^{96} \mathrm{Zr}$ nucleus including an analysis of the mixing between one-phonon $6^{+}$and two-phonon $\left[3_{1}^{-} \otimes 3_{1}^{-}\right]_{6}+$ configurations. It was shown that this mixing gives rise to two $6^{+}$states with enhanced and suppressed transition probabilities due to interference between one- and two-phonon configurations.

To conclude about the comparison of inelastic scattering data to $6^{+}$states with our structure calculations, we have to state that we obtain good agreement if we neglect two step reaction amplitudes. This is consistent with the fact that sequential double octupole transitions never had been observed unambiguously in inelastic scattering. On the other hand, to our knowledge, there is no reason to neglect these two step reaction amplitudes which are well established in the case of double quadrupole excitations. If one includes the two step amplitudes the agreement is only partial. One then has to conclude, that mixing of the $\left[3_{1}^{-} \otimes 3_{1}^{-}\right]_{6+}$ double octupole state with other $6^{+}$states, not included in the calculation, provides large effects resulting in a double octupole admixture to the lowest state with some strength more near to $20 \%$ than to $50 \%$ and a not yet identified spreading of the remaining strength over the higher lying states.

It should be added that when the present paper was intended to be submitted for publication a new measurement of the half-life of the $3_{1}^{-}$state in ${ }^{96} \mathrm{Zr}$ corresponding to the $B(E 3 \downarrow)$ value of $(47.1 \pm 4.7)$ W.u. appeared $\left.{ }^{26}\right)$. Reanalysis of inelastic ${ }^{6} \mathrm{Li}$ scattering data $\left.{ }^{22}\right)$ with this $B(E 3 \downarrow)$ value yields a good description $\left.{ }^{26}\right)$ of these data at $M_{n} / M_{p}=1.30$. Our calculations agree fairly well with these two newly deduced characteristics for the $3_{1}^{-}$state.

Useful discussions with Ph. Chomaz, P. Van Isacker, V. Khodel', M. Ploszajczak and E. Saperstein are gratefully acknowledged. One of the authors (S.F.) would like to thank kind hospitality of theory group at GANIL extended for him. 
The work was supported in part by the BMFT and DFG and by the International office in Karlsruhe. Two of us, S.F. and A.P., acknowledge the ISF and AIP foundation for the financial support during this research.

\section{Appendix A}

The central mean-field potentials related with main term of the density functional, eq. (2.5), are defined according to eq. (2.16):

$$
\begin{aligned}
U^{n(p)}= & \frac{\delta}{\delta \rho_{n, p}} \int d \vec{r} \varepsilon_{\text {main }} \\
& =\frac{\epsilon_{F}^{0}}{3}\left[a_{+}^{v}\left(2 x_{+} f_{+}^{v}+x_{+}^{2} f_{+}^{v \prime}\right)+a_{-}^{v}\left( \pm 2 x_{-} f_{-}^{v}+x_{-}^{2} f_{-}^{v \prime}\right)\right. \\
& \left.+2 a_{+}^{s}\left(x_{+} f_{+}^{s \prime}+f_{+}^{s}\right) \widetilde{f_{+}^{s} x_{+}}+2 a_{-}^{s}\left(x_{-} f_{-}^{s}{ }^{\prime} \pm f_{-}^{s}\right) \widetilde{f_{-}^{s} x_{-}}\right] .
\end{aligned}
$$

Here and below the upper (lower) sign refers to neutrons (protons) and prime denotes derivative with respect to $x_{+}$.

The Coulomb potential for protons reads

$$
U_{\text {coul }}^{p}=4 \pi e^{2}\left[\frac{1}{r} \int_{0}^{r} \rho^{p}(r) r^{2} d r+\int_{r}^{\infty} \rho^{p}(r) r d r\right]-e^{2}\left(\frac{3}{\pi} \rho^{p}(r)\right)^{1 / 3}
$$

The potentials generated by the spin-orbit and by the velocity spin-dependent interactions are [see eq. (2.12)]

$$
\begin{gathered}
U_{l s}^{i}=\frac{\delta}{\delta \rho_{l s}^{i}} \int d \vec{r} \varepsilon_{l s}[\hat{\rho}]=C_{0} r_{0}^{2} \sum_{k=n, p}\left[\kappa^{i k} \frac{1}{r} \frac{d \rho^{k}}{d r}+g_{1}^{i k} \frac{1}{2 r^{2}} \rho_{s l}^{k}\right](\vec{\sigma} \cdot \vec{l}) \\
U_{l s(c)}^{i}=\frac{\delta}{\delta \rho^{i}} \int d \vec{r} \varepsilon_{l s}[\hat{\rho}]=-C_{0} r_{0}^{2} \sum_{k=n, p} \kappa^{i k} \frac{1}{r^{2}} \frac{d\left(r \rho_{l s}^{k}\right)}{d r} .
\end{gathered}
$$

Here, $U_{l s(c)}^{i}$ are the contributions to the central mean-field potentials coming from $L S$-interaction. 


\section{Appendix B}

I. Zero-range $\delta$-forces from the "volume" part of the density functional can be written as

$$
\begin{aligned}
\mathcal{F}^{n n(p p)}= & \frac{C_{0}}{2}\left[a_{+}^{v}\left(f_{+}^{v}+2 x_{+} f_{+}^{v \prime}+\frac{x_{+}^{2}}{2} f_{+}^{v \prime \prime}\right)\right. \\
& \left.+a_{-}^{v}\left(f_{-}^{v} \pm 2 x_{-} f_{-}^{v \prime}+\frac{x_{-}^{2}}{2} f_{-}^{v \prime \prime}\right)\right], \\
\mathcal{F}^{n p, p n}= & \frac{C_{0}}{2}\left[a_{+}^{v}\left(f_{+}^{v}+2 x_{+} f_{+}^{v \prime}+\frac{x_{+}^{2}}{2} f_{+}^{v \prime \prime}\right)\right. \\
& \left.-a_{-}^{v}\left(f_{-}^{v}-\frac{x_{-}^{2}}{2} f_{-}^{v \prime \prime}\right)\right], \\
\mathcal{F}^{-}= & C_{0} a_{-}^{v} f_{-}^{v} .
\end{aligned}
$$

II. Zero-range $\delta$-forces from the "surface" part of the density functional, which correspond to the second graph plus the $\delta$-contribution from the first graph for $\mathcal{F}^{i k}$ in fig. 3 , are

$$
\begin{aligned}
\mathcal{F}^{n n(p p)}= & \frac{C_{0}}{2}\left[a_{+}^{s}\left\{\left(x_{+} f_{+}^{s}{ }^{\prime \prime}+2 f_{+}^{s}{ }^{\prime}\right) \widetilde{f_{+}^{s} x_{+}}-\left(x_{+} f_{+}^{s}{ }^{\prime}+f_{+}^{s}\right)^{2}\right\}\right. \\
& \left.+a_{-}^{s}\left\{\left(x_{-} f_{-}^{s}{ }^{\prime \prime} \pm 2 f_{-}^{s}\right) \widetilde{f_{-}^{s} x_{-}}-\left(x_{-} f_{-}^{s} \mp f_{-}^{s}\right)^{2}\right\}\right] \\
\mathcal{F}^{n p, p n}= & \frac{C_{0}}{2}\left[a_{+}^{s}\left\{\left(x_{+} f_{+}^{s}{ }^{\prime \prime}+2 f_{+}^{s}\right) \widetilde{f_{+}^{s} x_{+}}-\left(x_{+} f_{+}^{s}{ }^{\prime}+f_{+}^{s}\right)^{2}\right\}\right. \\
& \left.+a_{-}^{s}\left\{x_{-} f_{-}^{s}{ }^{\prime \prime} \widetilde{f_{-}^{s} x_{-}}-\left(x_{-} f_{-}^{s}\right)^{2}+\left(f_{-}^{s}\right)^{2}\right\}\right] \\
\mathcal{F}^{-}= & -C_{0} a_{-}^{s}\left(f_{-}^{s}\right)^{2} .
\end{aligned}
$$

III. Finite-range forces which correspond to the first graph for $\mathcal{F}^{i k}$ in fig. 3 are 
given by

$$
\begin{aligned}
\mathcal{F}^{n n(p p)}= & \frac{C_{0}}{2}\left[a _ { + } ^ { s } \left\{\left[x_{+}\left(\vec{r}_{1}\right) f_{+}^{s}{ }^{\prime}\left(\vec{r}_{1}\right)+f_{+}^{s}\left(\vec{r}_{1}\right)\right] V\left(\left|\vec{r}_{1}-\vec{r}_{2}\right|\right)\right.\right. \\
& \left.\times\left[x_{+}\left(\vec{r}_{2}\right) f_{+}^{s}{ }^{\prime}\left(\vec{r}_{2}\right)+f_{+}^{s}\left(\vec{r}_{2}\right)\right]\right\} \\
& +a_{-}^{s}\left\{\left[x_{-}\left(\vec{r}_{1}\right) f_{-}^{s}\left(\vec{r}_{1}\right) \pm f_{-}^{s}\left(\vec{r}_{1}\right)\right] V\left(\left|\vec{r}_{1}-\vec{r}_{2}\right|\right)\right. \\
& \left.\left.\times\left[x_{-}\left(\vec{r}_{2}\right) f_{-}^{s}{ }^{\prime}\left(\vec{r}_{2}\right) \pm f_{-}^{s}\left(\vec{r}_{2}\right)\right]\right\}\right], \\
\mathcal{F}^{n p, p n}= & \frac{C_{0}}{2}\left[a _ { + } ^ { s } \left\{\left[x_{+}\left(\vec{r}_{1}\right) f_{+}^{s}{ }^{\prime}\left(\vec{r}_{1}\right)+f_{+}^{s}\left(\vec{r}_{1}\right)\right] V\left(\left|\vec{r}_{1}-\vec{r}_{2}\right|\right)\right.\right. \\
& \left.\times\left[x_{+}\left(\vec{r}_{2}\right) f_{+}^{s}{ }^{\prime}\left(\vec{r}_{2}\right)+f_{+}^{s}\left(\vec{r}_{2}\right)\right]\right\} \\
& +a_{-}^{s}\left\{\left[x_{-}\left(\vec{r}_{1}\right) f_{-}^{s}{ }^{\prime}\left(\vec{r}_{1}\right) \pm f_{-}^{s}\left(\vec{r}_{1}\right)\right] V\left(\left|\vec{r}_{1}-\vec{r}_{2}\right|\right)\right. \\
& \left.\left.\times\left[x_{-}\left(\vec{r}_{2}\right) f_{-}^{s}\left(\vec{r}_{2}\right) \mp f_{-}^{s}\left(\vec{r}_{2}\right)\right]\right\}\right], \\
\mathcal{F}^{-}= & C_{0} a_{-}^{s} f_{-}^{s}\left(\vec{r}_{1}\right) V\left(\left|\vec{r}_{1}-\vec{r}_{2}\right|\right) f_{-}^{s}\left(\vec{r}_{2}\right) .
\end{aligned}
$$

Here $V(|\vec{r}|)$ is the Yukawa function defined as in eq. (2.8). For completness, we have shown also the charge-exchange interaction given by $\mathcal{F}^{-}=2 \frac{\delta^{2}}{\delta \rho_{-}^{2}} \int d \vec{r} \varepsilon_{\text {main }}$.

\section{Appendix C}

Effective three-body interaction amplitude $W^{i k l}$ is defined as the third functional derivative of the energy functional with respect to densities:

$$
W^{i k l}=\frac{\delta^{3}}{\delta \rho^{i} \delta \rho^{k} \delta \rho^{l}} \int d \vec{r} \varepsilon_{\operatorname{main}}
$$

where $\varepsilon_{\text {main }}$ is given by eq. (2.5). According to this density functional, the $W$ can be represented as a sum of four terms:

$$
W^{i k l}=W_{+}^{v, i k l}+W_{-}^{v, i k l}+W_{+}^{s, i k l}+W_{-}^{s, i k l}
$$

I. Local $\delta$-part of the three-body amplitude generated by the volume isoscalar term 
of the density functional, $\frac{2}{3} \epsilon_{F}^{0} \rho_{0} a_{+}^{v} x_{+}^{2} f_{+}^{v} \quad$ [see eq. (2.5)]:

$$
W_{+}^{v, i k l}=\frac{C_{0}}{4 \rho_{0}} a_{+}^{v}\left(3 f_{+}^{v \prime}+3 x_{+} f_{+}^{v \prime \prime}+\frac{1}{2} x_{+}^{2} f_{+}^{2 \prime \prime \prime}\right) \text {. }
$$

II. Local $\delta$-part of the three-tail amplitude generated by the volume isovector term of the density functional, $\frac{2}{3} \epsilon_{F}^{0} \rho_{0} a_{-}^{v} x_{-}^{2} f_{-}^{v}$ [see eq. (2.5)]:

$$
\begin{aligned}
W_{-}^{v, n n n} & =\frac{C_{0}}{4 \rho_{0}} a_{-}^{v}\left(3 f_{-}^{v \prime}+3 x_{-} f_{-}^{v \prime \prime}+\frac{1}{2} x_{-}^{2} f_{-}^{v \prime \prime \prime}\right), \\
W_{-}^{v, n n p, n p n, p n n} & =\frac{C_{0}}{4 \rho_{0}} a_{-}^{v}\left(-f_{-}^{v \prime}+x_{-} f_{-}^{v \prime \prime}+\frac{1}{2} x_{-}^{2} f_{-}^{v \prime \prime \prime}\right), \\
W_{-}^{v, n p p, p n p, p p n} & =\frac{C_{0}}{4 \rho_{0}} a_{-}^{v}\left(-f_{-}^{v \prime}-x_{-} f_{-}^{v \prime \prime}+\frac{1}{2} x_{-}^{2} f_{-}^{v \prime \prime \prime}\right), \\
W_{-}^{v, p p p} & =\frac{C_{0}}{4 \rho_{0}} a_{-}^{v}\left(3 f_{-}^{v \prime}-3 x_{-} f_{-}^{v \prime \prime}+\frac{1}{2} x_{-}^{2} f_{-}^{v \prime \prime \prime}\right) .
\end{aligned}
$$

III. The part of the three-body amplitude generated by the surface isoscalar term of the density functional, $\frac{2}{3} \epsilon_{F}^{0} \rho_{0} a_{+}^{s} x_{+} f_{+}^{s} \widetilde{f_{+}^{s} x}+\quad$ [see eq. (2.5)]:

$$
W_{+}^{s, i k l}=W_{\delta,+}^{s, i k l}+W_{r,+}^{s, i k l}
$$

Here the local term, which corresponds to the last graph in fig. 3 plus the $\delta$-contributions from three preceeding graphs, is given by

$$
W_{\delta,+}^{s, i k l}=\frac{C_{0}}{4 \rho_{0}} a_{+}^{s}\left(\phi_{+}{ }^{\prime \prime \prime} \widetilde{\phi_{+}}-3 \phi_{+}{ }^{\prime \prime} \phi_{+}{ }^{\prime}\right),
$$

and the finite-range term, which corresponds to the sum of three first graphs in fig. 3 for the amplitude $W^{i k l}$, can be written as

$$
\begin{aligned}
W_{r,+}^{s, i k l}= & \frac{C_{0}}{4 \rho_{0}} a_{+}^{s}\left[\phi_{+}^{\prime \prime(i l)}\left(\vec{r}_{1}\right) V\left(\left|\vec{r}_{1}-\vec{r}_{2}\right|\right) \phi_{+}^{\prime(k)}\left(\vec{r}_{2}\right)\right. \\
& \left.+\phi_{+}^{\prime(i)}\left(\vec{r}_{1}\right) V\left(\left|\vec{r}_{1}-\vec{r}_{2}\right|\right) \phi_{+}^{\prime \prime(k l)}\left(\vec{r}_{2}\right)+\phi_{+}^{\prime \prime(i k)}\left(\vec{r}_{1}\right) V\left(\left|\vec{r}_{1}-\vec{r}_{2}\right|\right) \phi_{+}^{\prime(l)}\left(\vec{r}_{2}\right)\right],
\end{aligned}
$$

where $\phi_{+}=x_{+} f_{+}^{s}$. It should be noticed that right-hand side of eq. (C.6) does not contain explicitly the indecies $i k l$ whereas they are kept in eq. (C.7) to show a possible 
dependence on the angular momenta of the external particle-hole (phonon) pairs. This means that each index corresponds not only to a derivative with respect to neutron or proton density but also to a certain multipolarity.

The part of the three-body amplitude generated by the surface isovector term of the density functional, $\frac{2}{3} \epsilon_{F}^{0} \rho_{0} a_{-}^{s} x_{-} f_{-}^{s} \widetilde{f_{-}^{s} x_{-}} \quad$ [see eq. (2.5)]:

$$
W_{-}^{s, i k l}=W_{\delta,-}^{s, i k l}+W_{r,-}^{s, i k l}
$$

Here the local term is given by

$$
\begin{aligned}
W_{\delta,-}^{s, i k l}=\frac{C_{0}}{4 \rho_{0}} a_{-}^{s} & {\left[\phi_{-}{ }^{\prime \prime \prime(i k l)} \widetilde{\phi_{-}}-\phi_{-}{ }^{\prime \prime(i l)} \phi_{-}{ }^{\prime(k)}\right.} \\
& \left.-\phi_{-}{ }^{\prime(i)} \phi_{-}{ }^{\prime \prime(k l)}-\phi_{-}{ }^{\prime \prime(i k)} \phi_{-}{ }^{\prime}(l)\right],
\end{aligned}
$$

and the finite-range term can written as

$$
\begin{aligned}
W_{r,-}^{s, i k l}=\frac{C_{0}}{4 \rho_{0}} a_{-}^{s} & {\left[\phi_{-}{ }^{\prime \prime(i l)}\left(\vec{r}_{1}\right) V\left(\left|\vec{r}_{1}-\vec{r}_{2}\right|\right) \phi_{-}{ }^{\prime(k)}\left(\vec{r}_{2}\right)\right.} \\
& +\phi_{-}{ }^{\prime(i)}\left(\vec{r}_{1}\right) V\left(\left|\vec{r}_{1}-\vec{r}_{2}\right|\right) \phi_{-}{ }^{\prime \prime(k l)}\left(\vec{r}_{2}\right) \\
& \left.+\phi_{-}{ }^{\prime \prime(i k)}\left(\vec{r}_{1}\right) V\left(\left|\vec{r}_{1}-\vec{r}_{2}\right|\right) \phi_{-}{ }^{\prime(l)}\left(\vec{r}_{2}\right)\right]
\end{aligned}
$$

where $\phi_{-}=x_{-} f_{-}^{s}$ 


\section{Figure captions}

Fig. 1. Graphical representation of eq. (3.6) for one-phonon excitation amplitude.

Fig. 2. Graphical representations of eq. (4.7) for the two-phonon excitation amplitude and of eq. (4.2) for the matrix element of excitation of two-phonon state in the external field $V_{0}$.

Fig. 3. Graphical representation of interaction energy density, mean field potentials and effective two- and three- body interactions. Dashed lines correspond to both local and finite-range forces, full circles contain density-dependent functions and ticks denote functional derivatives with respect to corresponding densities while indexes $i, k, l$ reffering to neutrons or protons may incorporate also a dependence on angular momenta.

Fig. 4. Graphical representation of the interaction matrix element $V_{1 p h-2 p h}$, eq. (5.2), between one-phonon $6^{+}$state and two-phonon $\left[3^{-} \otimes 3^{-}\right]_{6}+$ state.

Fig. 5 .

Fig.5a Calculated ground state density distributions for neutrons and protons in ${ }^{96} \mathrm{Zr}$.

Fig.5b Full curve: microscopically calculated neutron transition density to the one-phonon $3_{1}^{-}$state in ${ }^{96} \mathrm{Zr}$. Dashed curve: collective model prediction.

Fig.5c Full curve: microscopically calculated neutron transition density to the one-phonon $6^{+}$state in ${ }^{96} \mathrm{Zr}$. Dashed curve: collective model prediction.

Fig.5d Full curve: microscopically calculated neutron transition density to the two-phonon $6^{+}$state in ${ }^{96} \mathrm{Zr}$. Dashed curves: collective model predictions.

Fig.5e Microscopically calculated neutron transition densities for the $6^{+}$states in ${ }^{96} \mathrm{Zr}$ for pure one-phonon (1), pure two-phonon (2) and one- and two-phonon mixed transitions according to eqs.(5.4) (3 and 4). 
Fig. 6

Fig.6a $22 \mathrm{MeV}$ (d,d') data of the two $6^{+}$states with DWBA and coupled channels calculations. Full curves: DWBA calculation for the $6^{+}$states at $3.483 \mathrm{MeV}$ and $3.772 \mathrm{MeV}$ with $B(I 6)=10.9$ W.u. and $B(I 6)=0.60$ W.u., respectively, according to ref. ${ }^{1}$ ). Dashed curves: Pure two-step two-phonon coupled channels calculation with a $B(I 3)$ of 39.2 W.u. Dotted curves: coupled channels calculation for the mixing of a pure two-step two-phonon excitation with a $B(I 3)$ of 39.2 W.u. with a one-step excitation having an effective direct excitation strength of $B(I 6)=10.8$ W.u. and 0.034 W.u., respectively, as predicted by this work. The mixing angle is $+45^{\circ}$.

Fig.6b The full curves and dashed curves are the same as in fig. 6a. The dotted curve is calculated as in fig. $6 \mathrm{a}$ but with a mixing angle of $135^{\circ}$. 


\section{References}

1. D. Hofer, M. Bisenberger, R. Hertenberger, H. Kader, H. J. Maier, E. MüllerZanotti, P. Schiemenz, G. Graw, P. Maier-Komor, G. Molnár, W. Unkelbach, J. Hebenstreit, H. Ohm, D. Paul, P. von Rossen and M. Fujiwara, Nucl. Phys. A551 (1993) 173

2. O. A. Rosso, W. Unkelbach and G. Molnár, Nucl. Phys. A563 (1993) 74

3. E. E. Saperstein, A. V. Tolokonnikov and S. A. Fayans, Preprint IAE-2571 (in Russian), Moscow (1975); I. N. Borzov and S. A. Fayans, Preprint IPPE-1129 (in Russian), Obninsk (1981)

4. S. Shlomo and G. Bertsch, Nucl. Phys. A243 (1975) 507

5. E. E. Saperstein, S. A. Fayans and V. A. Khodel', Fiz. Elem. Chastits At. Yadra 9 (1978) 221 [Sov. J. Part. Nucl. 9 (1978) 91]; S. A. Fayans, E. E. Saperstein and V. A. Khodel', Nucl. Phys. A317 (1979) 424; V. A. Khodel' and E. E. Saperstein, Nucl. Phys. A348 (1980) 261

6. P. Hohenberg and W. Kohn, Phys. Rev. B136 (1964) 864

7. W. Kohn and L. Sham, Phys. Rev. A140 (1965) 1133

8. V. A. Khodel' and E. E. Saperstein, Phys. Reports 92 (1982) 183

9. D. Vautherin and D. Brink, Phys. Rev. C5 (1972) 626

10. A. V. Smirnov, S. V. Tolokonnikov and S. A. Fayans, Yad. Fiz. 48 (1988) 1661 [Sov. J. Nucl. Phys. 48 (1988) 995]

11. A. B. Migdal, Teoriya konechnykh fermi-sistem i svoistva atomnykh yader [Theory of Finite Fermi Systems and Properties of Atomic Nuclei], 2nd Edition, Moscow, Nauka, 1983 
12. S. A. Fayans, in preparation

13. S. A. Fayans, E. L. Trykov and D. Zawischa, Nucl. Phys. A, in press

14. W. Kim, B. L. Miller, J. R. Calarco, L. S. Cardman, J. P. Connelly, S. A. Fayans, B. Frois, D. Goutte, J. H. Heisenberg, F. W. Hersman, V. Meot, T. E. Milliman, P. Mueller, C. N. Papanicolas, A. P. Platonov, V. Yu. Ponomarev and J. E. Wise, Phys. Rev. C45 (1992) 2290

15. J. E. Wise, J. P. Connelly, F. W. Hersman, J. H. Heisenberg, W. Kim, M. Leuschner, S. A. Fayans, A. P. Platonov, E. E. Saperstein and V. Yu. Ponomarev, Phys. Rev. C45 (1992) 2701

16. W. Kim, J. P. Connely, J. H. Heisenberg, F. W. Hersman, T. E. Milliman, J. E. Wise, C. N. Papanicolas, S. A. Fayans and A. P. Platonov, Phys. Rev. C46 (1992) 1656

17. V. Yu. Ponomarev, W. T. A. Borghols, S. A. Fayans, M. N. Harakeh, C. W. de Jager, J. B. van der Laan, A. P. Platonov, H. de Vries and S. Y. van der Werf, Nucl. Phys. A549 (1992) 180

18. J. E. Wise, J. R. Calarco, J. P.Connely, S. A. Fayans, F. W. Hersman, J. H. Heisenberg, R. S. Hicks, W. Kim, T. E. Milliman, R. A. Miskimen, G. A. Peterson, A. P. Platonov, E. E. Saperstein and R. P. Singhal, Phys. Rev. C47 (1993) 2539

19. A. P. Platonov and E. E. Saperstein, Nucl. Phys. A486 (1988) 63

20. S. T. Belyaev, Zh. Eksp. Teor. Fiz. 39 (1960) 1387 [Sov. Phys. JETP 12 (1961) $968]$

21. S. A. Fayans and V. A. Khodel', Pis'ma Zh. Eksp. Theor. Fiz. 17, 633 (1973) [JETP Letters 17 (1973) 444]

22. D. J. Horen, R. L. Auble, J. Gomez del Campo, R. L. Varner, J. R. Beene, 
G. R. Satchler, B. Lund, V. R. Brown, P. L. Anthony and V. A. Madsen, Phys. Lett. B296 (1992) 18; D. J. Horen, R. L. Auble, J. Gomez del Campo, G. R. Satchler, R. L. Varner, J. R. Beene, B. Lund, V. R. Brown, P. L. Anthony and V. A. Madsen, Phys. Rev. C47 (1993) 629

23. G. Molnár, T. Belgya, B. Fazekas, Á. Veres, S. W. Yates, E. W. Kleppinger, R. A. Gatenby, R. Julin, J. Kumpulainen, A. Passoja and E. Verho, Nucl. Phys. A500 (1989) 43

24. H. Ohm, M. Liang, G. Molnár, S. Raman, K. Sistemich and W. Unkelbach, Phys. Lett. B241 (1990) 472

25. H. Mach, S. Ćwiok, W. Nazarewicz, B. Fogelberg, M. Moszyński, J. Winger and R. L. Gill, Phys. Rev. C42 (1990) R811

26. D. J. Horen, R. L. Auble, G. R. Satchler, J. R. Beene, I. Y. Lee, C. Y. Wu, D. Cline, M. Devlin, R. Ibbotson and M. W. Simon, Phys. Rev. C48 (1993) R2131 
Table 1. Calculated transition moments and RMS radii for some states in ${ }^{96} \mathrm{Zr}$ (for the $6^{+}$states $M_{n}$ and $M_{p}$ should be multiplied by $10^{3}$ )

\begin{tabular}{|c|c|c|c|c|c|c|c|c|}
\hline & $\begin{array}{c}M_{n} \\
{\left[\mathrm{fm}^{\lambda}\right]} \\
\end{array}$ & $\begin{array}{l}M_{p} \\
{\left[\mathrm{fm}^{\lambda}\right]} \\
\end{array}$ & $\frac{M_{n}}{M_{p}}$ & $\begin{array}{l}B(E \lambda \downarrow) \\
{[\mathrm{W} . \mathrm{u} .]}\end{array}$ & $\begin{array}{l}B(I \lambda) \\
{[\text { W.u. }]}\end{array}$ & $\begin{array}{c}\sqrt{\left\langle r_{n}^{2}\right\rangle} \\
{[\mathrm{fm}]}\end{array}$ & $\begin{array}{l}\sqrt{\left\langle r_{p}^{2}\right\rangle} \\
{[\mathrm{fm}]}\end{array}$ & $\begin{array}{l}\sqrt{\left\langle r_{I S}^{2}\right\rangle} \\
{[\mathrm{fm}]}\end{array}$ \\
\hline $0_{1}^{+}(g . s)$. & & & & & & 4.38 & 4.20 & 4.31 \\
\hline $2_{1}^{+}$ & 18.7 & 10.2 & 1.84 & 3.98 & 5.57 & 5.59 & 5.26 & 5.47 \\
\hline $3_{1}^{-}$ & 204 & 147 & 1.39 & 39.5 & 39.2 & 5.57 & 5.33 & 5.46 \\
\hline $6^{+}(1 p h)^{\prime}$ & 12.3 & 2.88 & 4.27 & 1.24 & 5.98 & 6.19 & 5.46 & 5.97 \\
\hline $6+(2 p h)$ & 8.50 & 5.12 & 1.66 & 3.91 & 4.80 & 6.43 & 6.10 & 6.28 \\
\hline $6_{(+)}^{+}$ & 14.7 & 5.66 & 2.60 & 4.78 & 10.8 & 6.27 & 5.81 & 6.11 \\
\hline $6_{(-)}^{+}$ & -2.73 & 1.58 & -1.72 & 0.37 & 0.034 & 5.84 & 10.8 & 5.01 \\
\hline
\end{tabular}



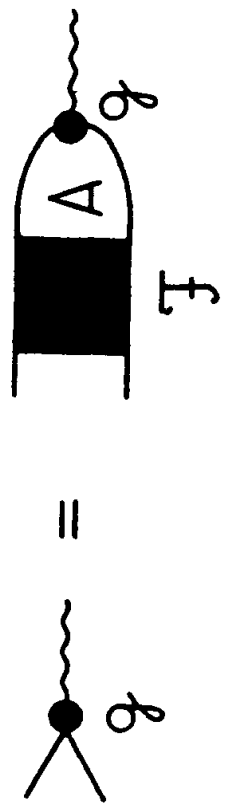

Di工 


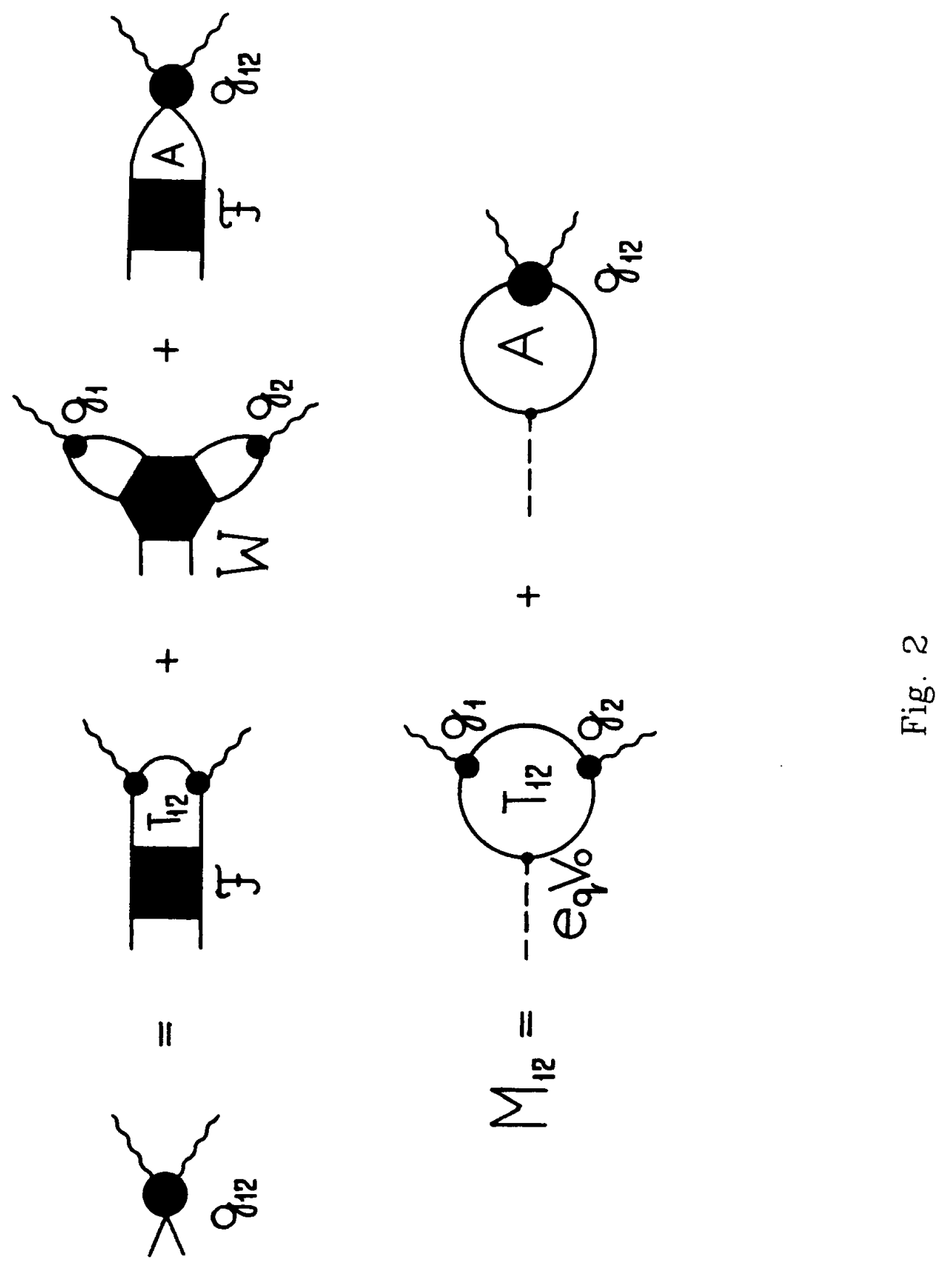




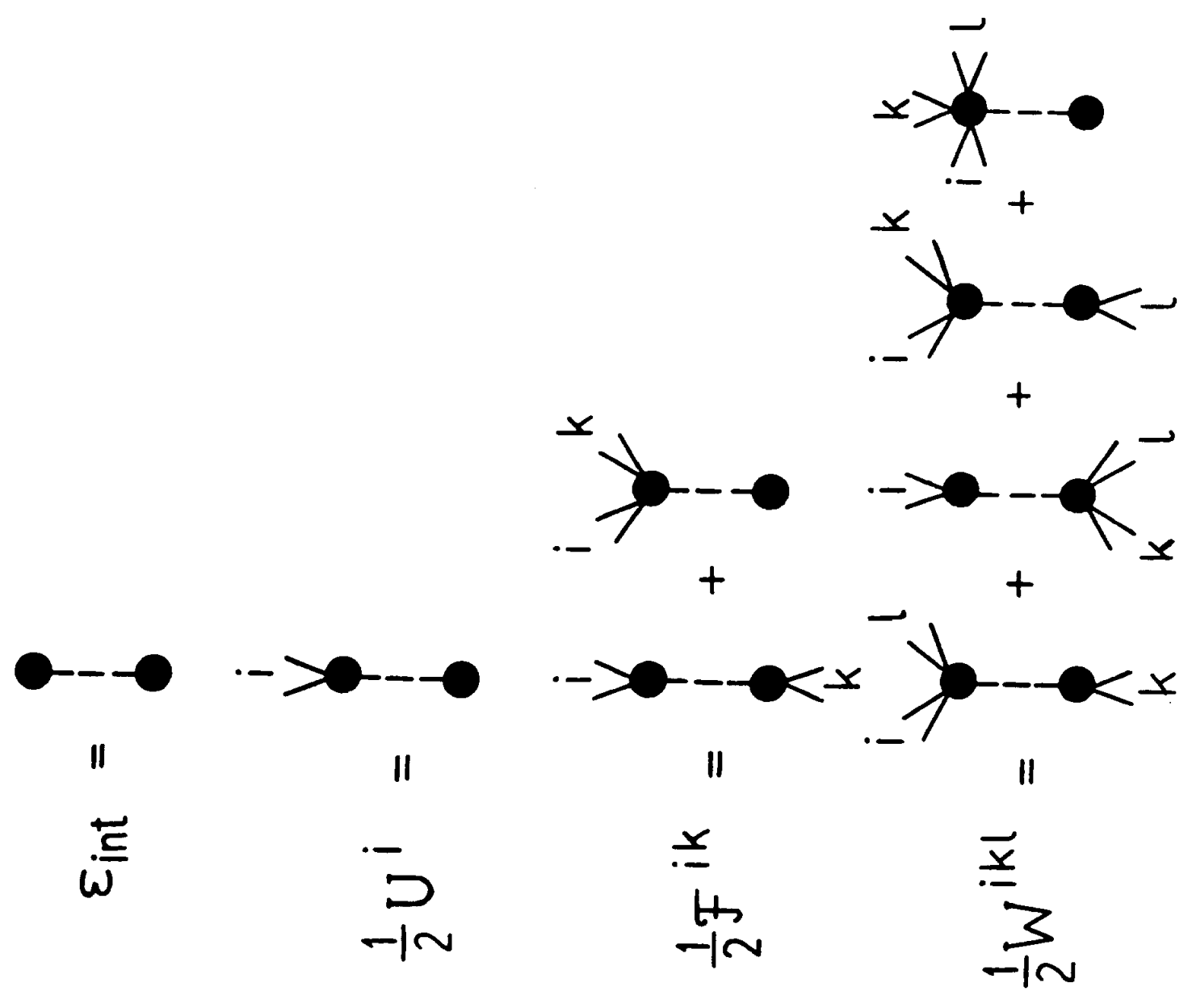

$m$

is 


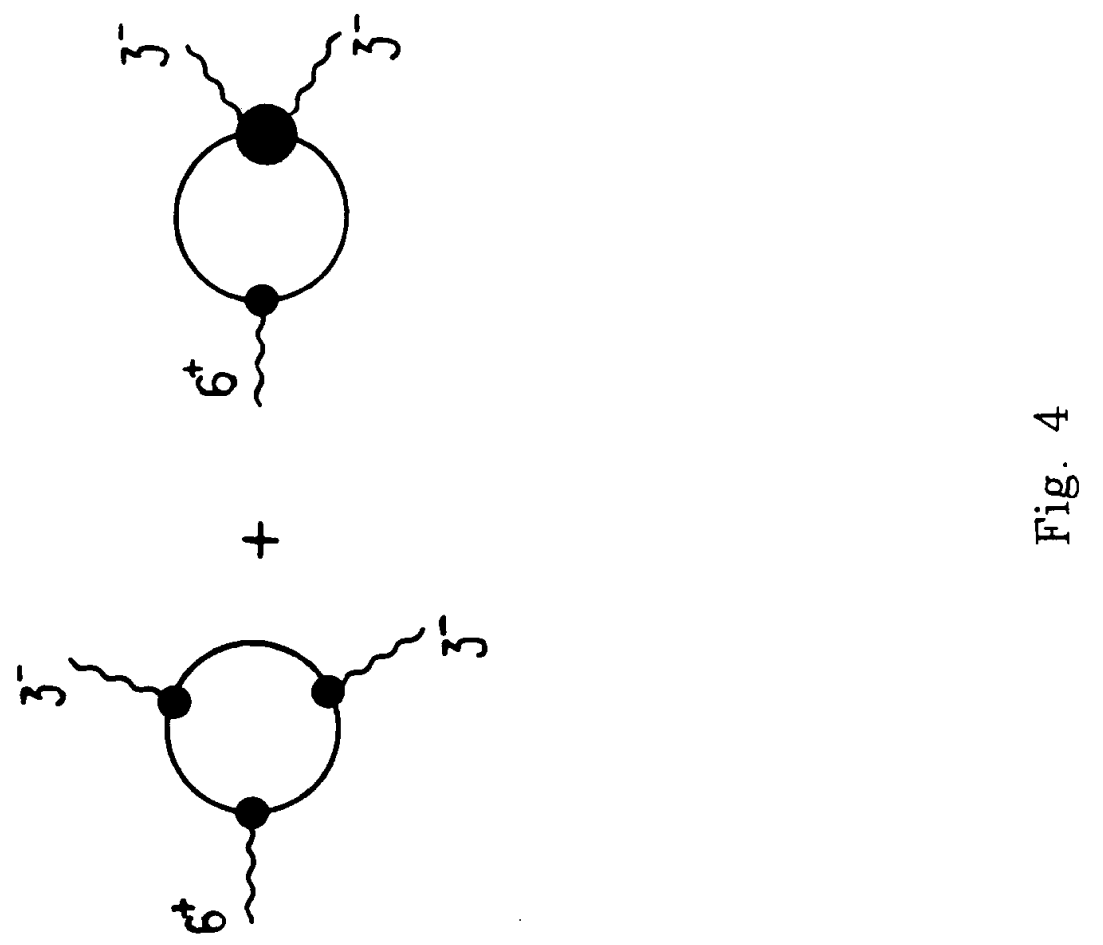




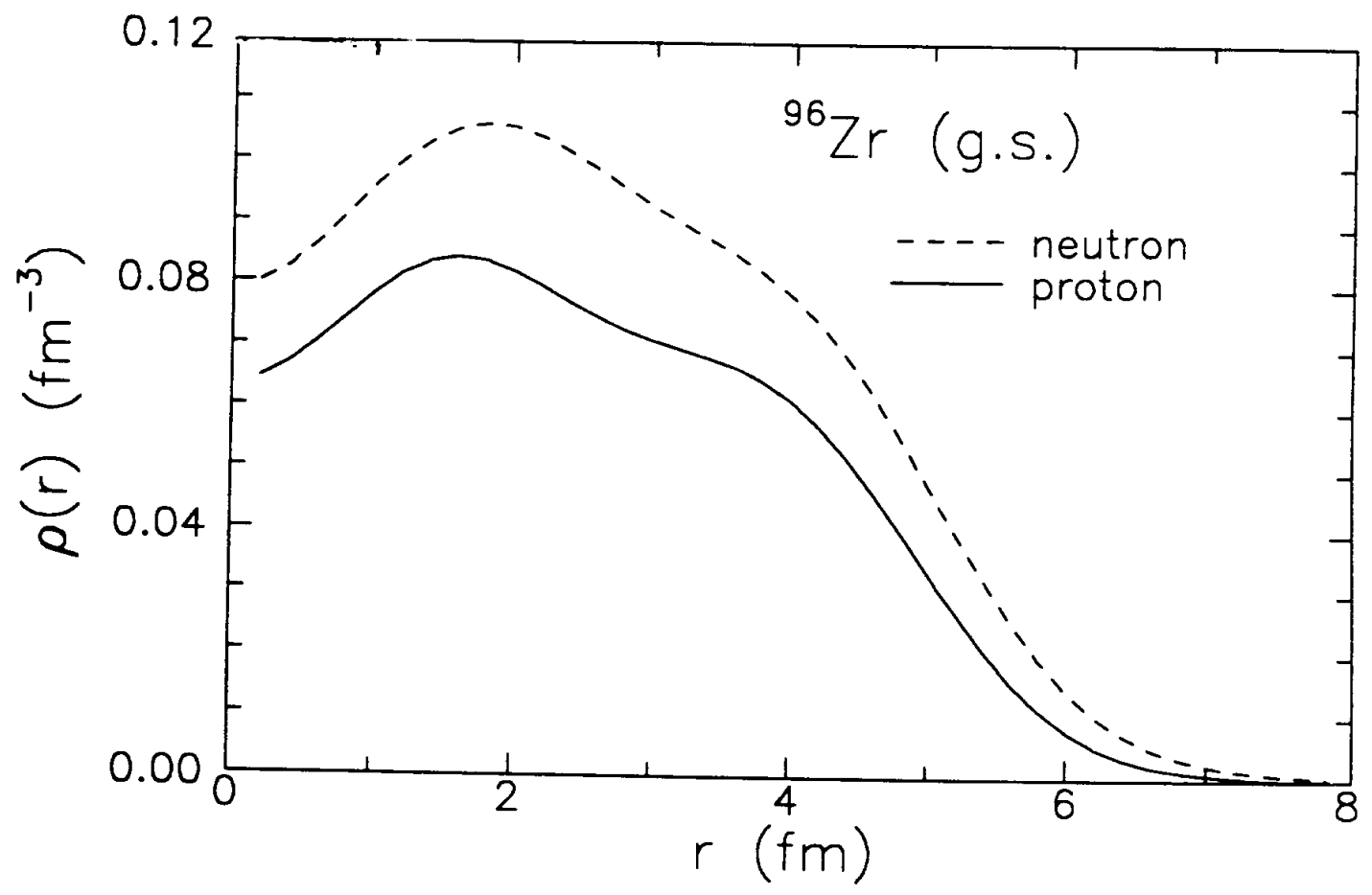

Fig. 5a 


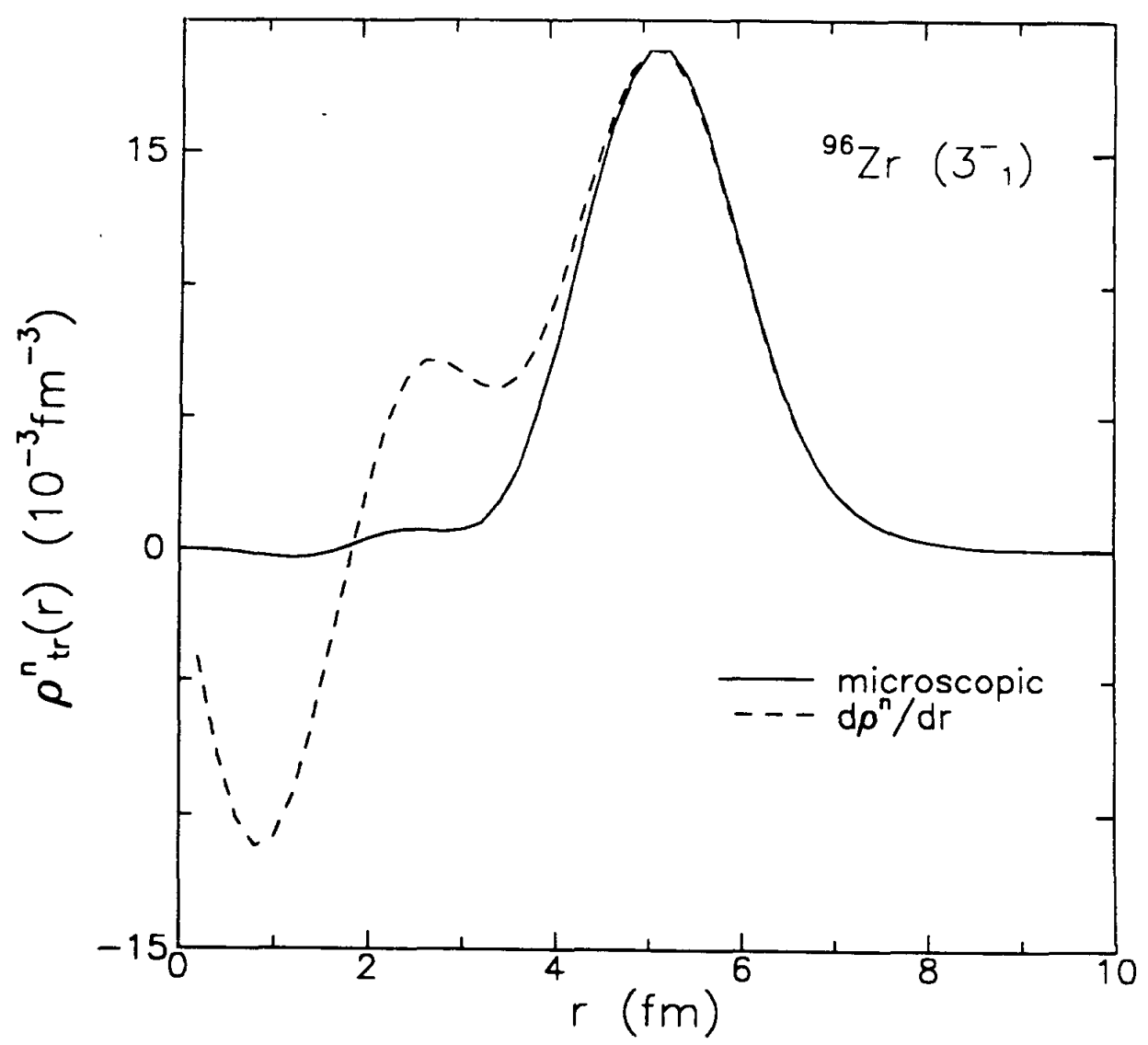

Fig. 5b 


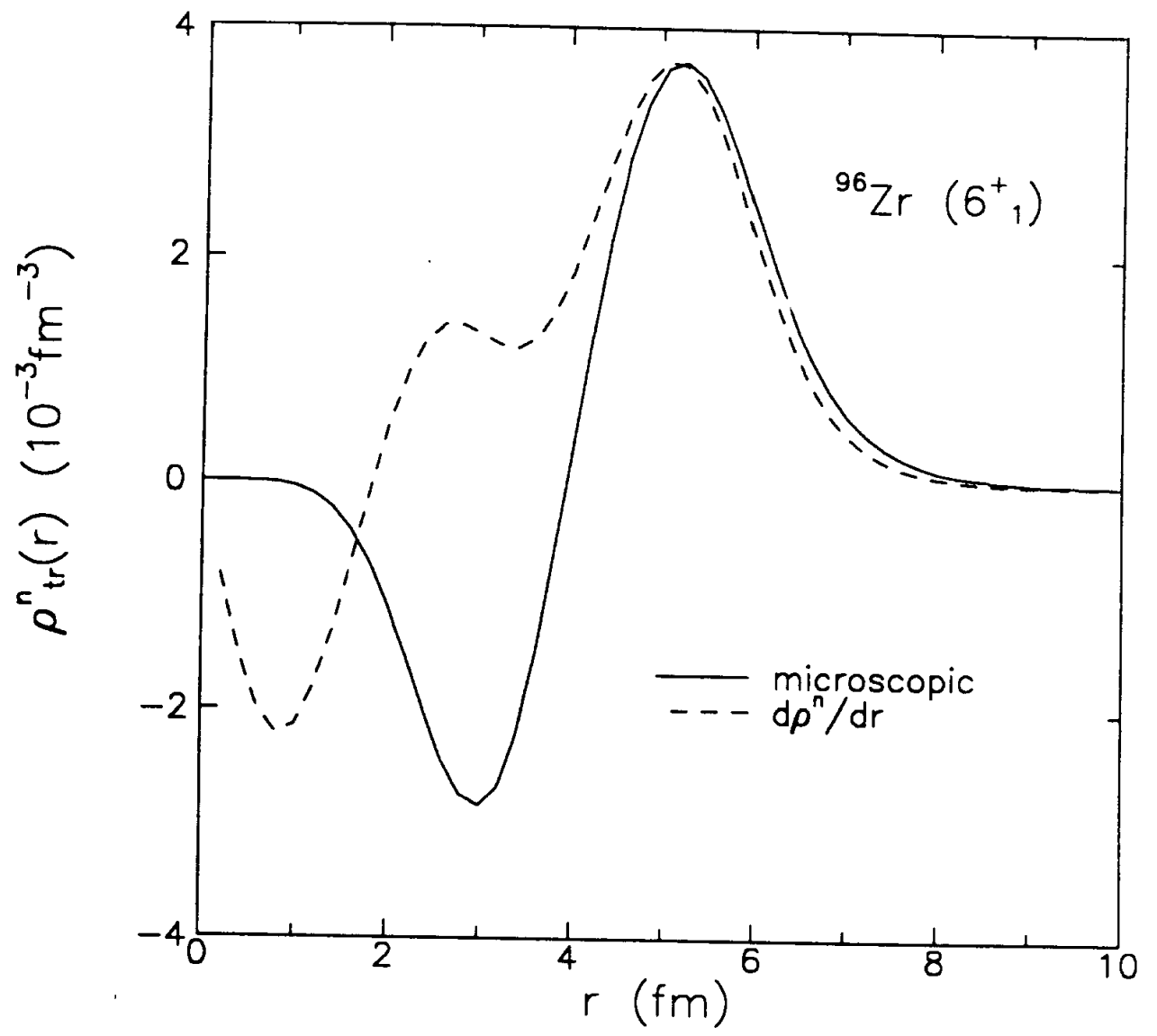

Fig. 5c 


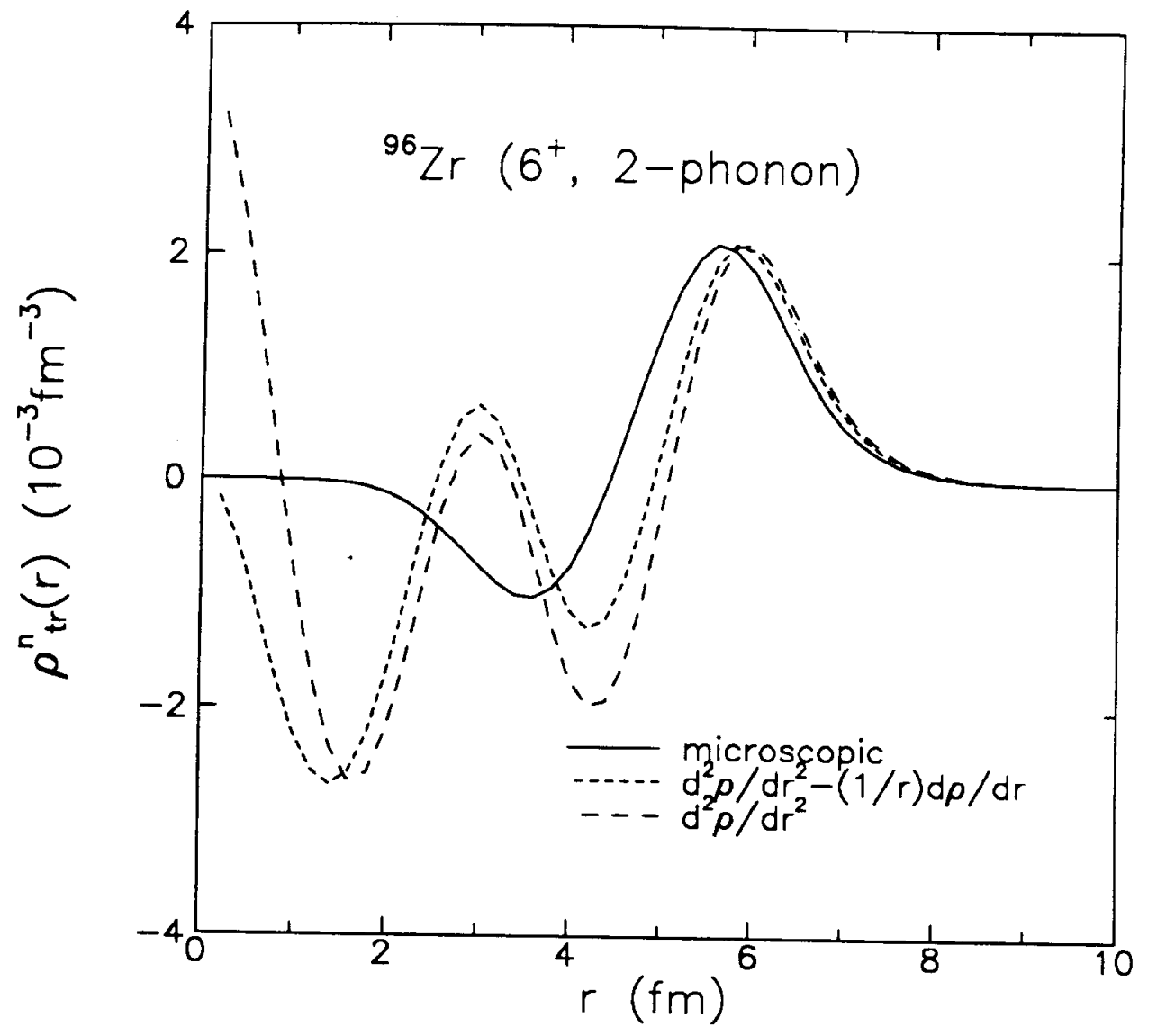

Fig. 5d 


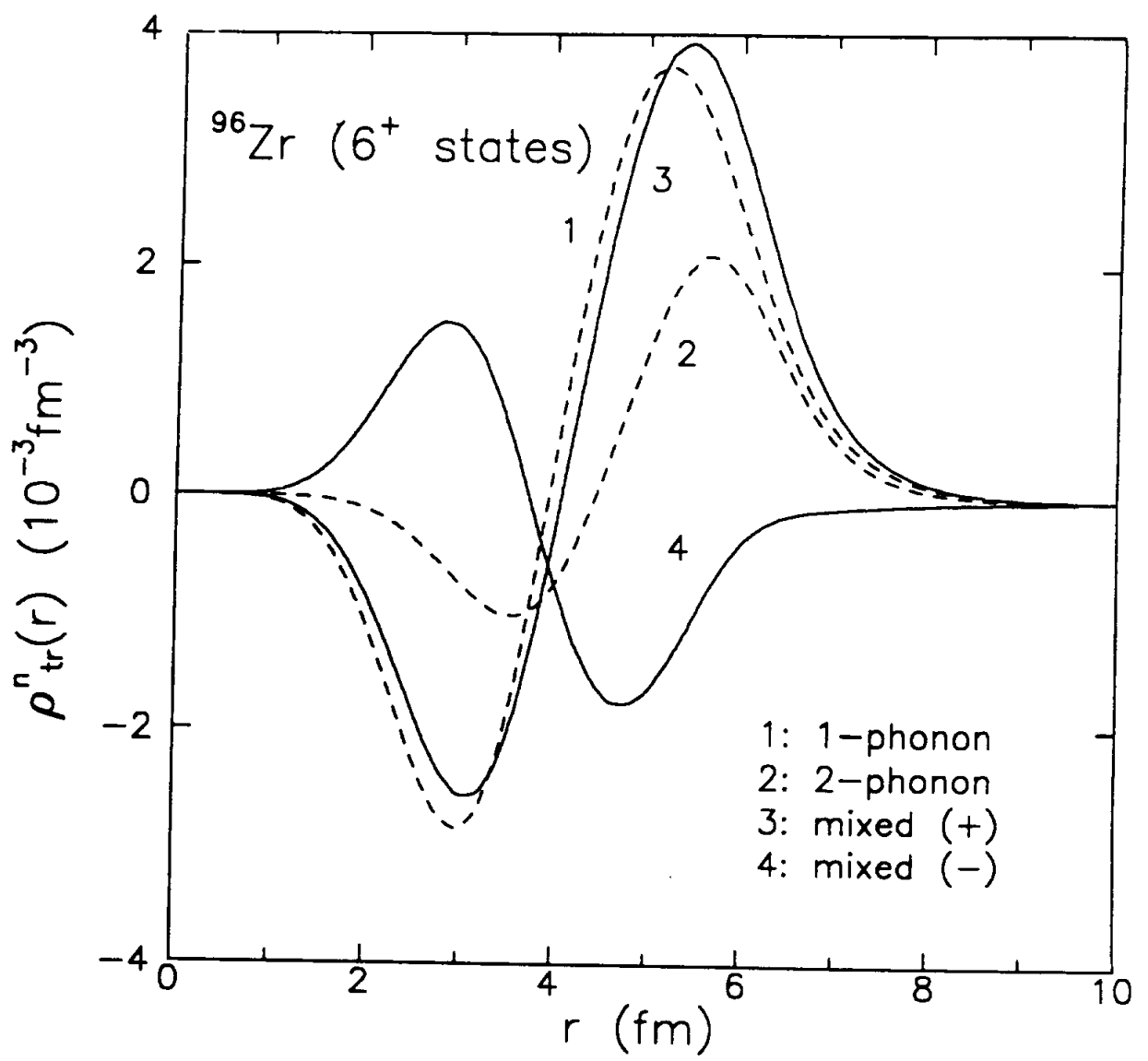

Fig. 5e 


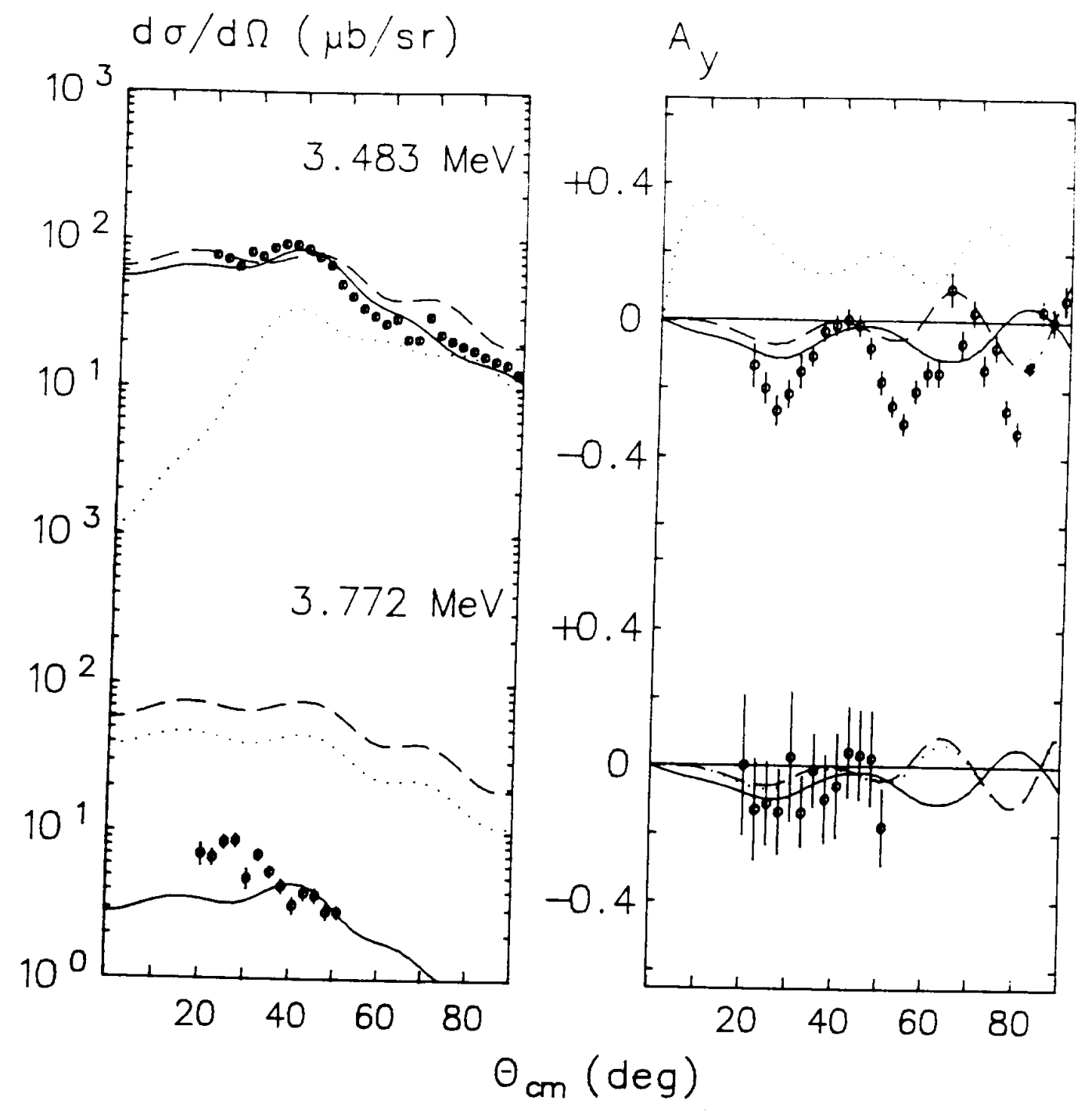

Fig. 6a 


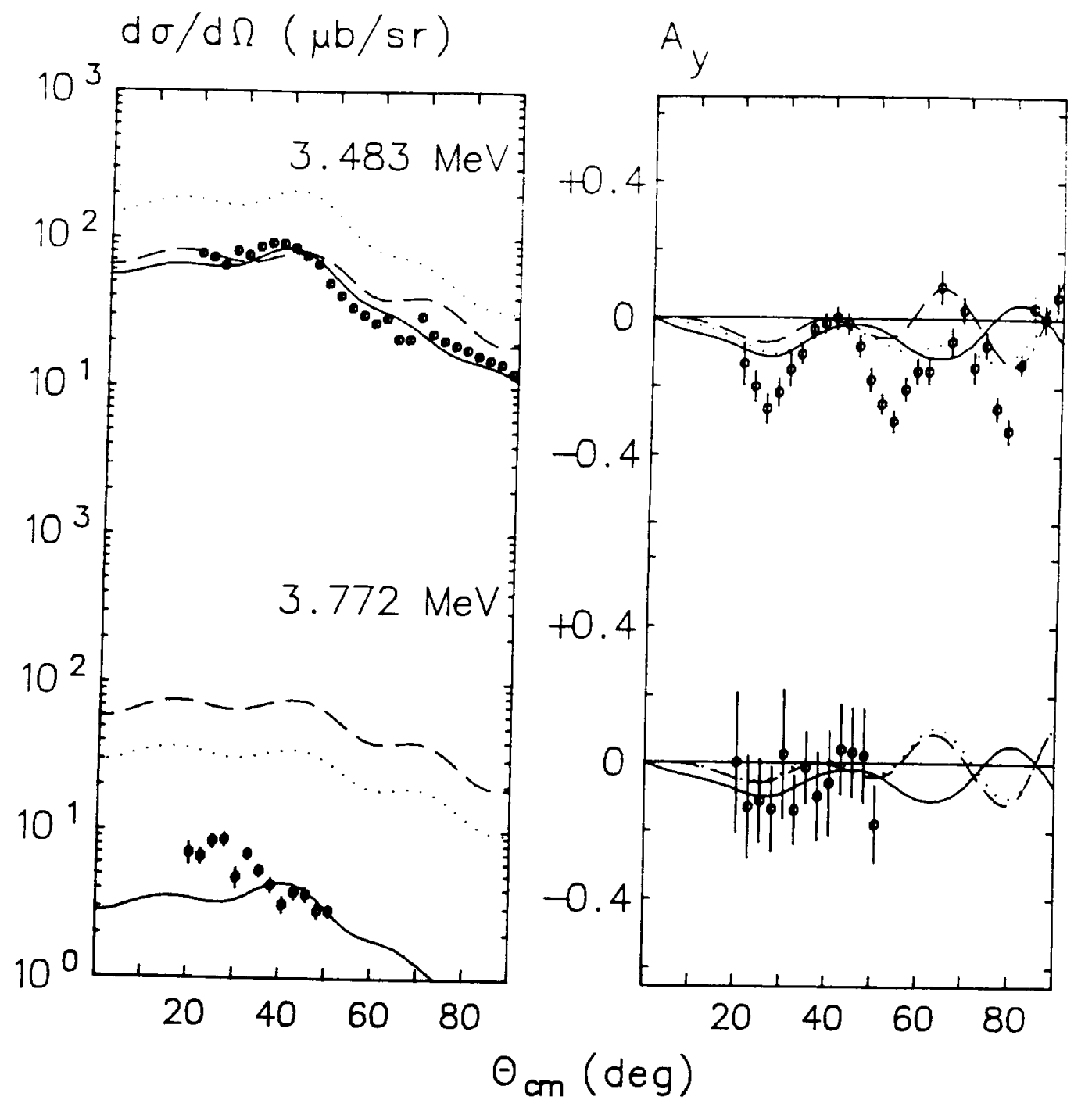

Fig. 6b 
